Noname manuscript No.

(will be inserted by the editor)

\title{
Air entrainment modeling in the SPH method: a two-phase mixture formulation with open boundaries
}

\author{
Thomas Fonty · Martin Ferrand • Agnès \\ Leroy · Damien Violeau
}

Received: date / Accepted: date

\begin{abstract}
Air entrainment within water is a common feature of flows over hydraulic works - spill over a dam, wave breaking on a dike, etc. - and its accurate modeling is a key to better design such structures. The Smoothed Particle Hydrodynamics (SPH) method appears as a natural way to model such highly distorted flows. To avoid computationally prohibitive costs related to the full discretization of bubbles or drops, a mixture model for high density ratio flows relying on a volume-based formulation with relative velocity between phases has first been developed and validated in $[21,22]$. Instead of having a once and for all assigned phase as in multifluid SPH, each particle now carries both phases through their respective volume fractions. In the present work, in order to handle practical air entrainment application cases, the open boundary formulation described in [19] is adapted to this mixture model. Then, after introducing turbulence through a $k-\epsilon$ model, a specific closure on the air bubbles relative velocity is proposed including a Stokesian drag term and turbulent diffusion. This model is then applied to two cases of air entrainment: a stepped spillway for interfacial aeration and a plunging jet for local aeration. Finally a 3D industrial test case of discharge-control structure at the La Coche power plant (France) is considered. While valuable insights are obtained for the volume fraction field, further investigations are required to improve the modeling of the flow dynamics.
\end{abstract}

This work was funded by EDF R\&D and the French Research Agency (CIFRE grant agreement \#2016-0362).

T. Fonty

EDF R\&D

6, quai Watier 78400 Chatou, France

E-mail: thomas.fonty@edf.fr

M. Ferrand

EDF R\&D - CEREA (joint Lab between Ecole des Ponts ParisTech and EDF R\&D)

A. Leroy

RTE

D. Violeau

EDF R\&D - LHSV (joint Lab between Ecole des Ponts ParisTech, EDF R\&D and Cerema) 
Keywords SPH $\cdot$ air entrainment $\cdot$ turbulence $\cdot$ hydraulic works $\cdot$ two-phase flows

\section{Introduction}

The entrainment and advection-diffusion of air bubbles within flowing water is pivotal for the design of a wide range of engineering applications from open channels situations (e.g. hydraulic works such as spillways, oxygenation of the flows) to closed conduit configurations (e.g. pipes in hydraulic plants) and chemical and environmental systems (e.g. reactor tanks). The multicomponent and multiscale nature of these flows makes their modeling challenging. The air presence indeed modifies the flow dynamics, the turbulence, and can lead to a wide variety of twophase regimes. The air entrainment in itself involves many mechanisms: air-suction at the free surface, air bubbles distribution according to their size, air bubble motion dynamics, interactions with turbulence... Extensive experimental studies have been carried out for hydraulic structures [10]. Regarding the role of surface tension and turbulence in bubble entrainment dynamics, it is complex to represent satisfactorily these flows in physical scale models of a laboratory. Their numerical modeling also remains complex, to follow macroscopically the deformation of the interface and simultaneously the generation, transport and interactions of bubbles and droplets. The resolution of instantaneous equations proves to be computationally prohibitively expensive. To overcome this limitation and handle practical problems, multiphase averaged models were developed in the past decades [31]: a volume fraction representation allow one to track phases while the interface is no longer followed explicitly. Among those averaged approaches, the mixture model considers the flow as a single-fluid with one continuity and one momentum equation for mixture quantities, complemented by a mass conservation equation for one phase. The relative velocity between phases, closed by a physics-dependent expression, introduces additional terms. This approach has been applied to airwater cases such as plunging jets [50] or stepped spillways $[45,42]$ within the finite volume numerical framework. Due to the strong deformations of the interfaces within the air-water flows, using a Lagrangian approach such as the Smoothed Particle Hydrodynamics ( $\mathrm{SPH}$ ) method is enticing. Mixture models have already been tested in SPH in case of low to moderate density ratio flows: [15], followed by [24], detailed a mixture model with diffusion of phases between particles; fluid mixtures were simulated in the graphics community by [51,64]; gas/dust mixtures were studied in the astrophysical framework by $[48,49]$ with a relative velocity computed using a drag law; water-sediment mixture were investigated $[3,58]$. SPH particles are no longer assigned to a specific phase: each individual particle carries the different phases with their respective volume fractions and are moved with a unique mixture velocity field. In this work, we aim at testing the performances of the SPH mixture model developed in $[21,22]$ for air-water flows. Nevertheless, this modeling requires to tackle several questions, addressed in this work:

- High density ratio between air and water: most of the cited mixture models rely on a mass-based formulation for the velocity: to handle high density ratio flows more easily, we have made here the choice to work with a volume-based formulation that simplifies some terms of the system of equations ruling the 
two-component system. However additional numerical treatment might be required for the discrete momentum equation resolution.

- Turbulence modeling: turbulence is often at the core of the entrainment phenomenon and makes it a full multi-scale problem. It deforms the air-water interface and the consequent irregularities can trap air bubbles. The downwards vertical velocity generated by the turbulence, if strong enough to exceed the terminal velocity caused by buoyancy of air bubbles, leads to diffusion of air in the bulk of the fluid. In this work, the standard $k-\epsilon$ model is considered as first approach.

- Relative velocity closure: it encompasses the two-component behavior of the flow and is intimately linked to the previous item relative to turbulence. If it enables the versatility of the mixture model (work with either air-water or water-sediment flows), this closure remains flow-dependent. We tried to keep a sufficiently general closure for the type of flow considered in this work.

- Open boundaries: most air entrainment cases involve inlet and outlet. Important work was performed recently on open boundaries in SPH, most of them involving a buffer layer. However, few works considered multifluid open boundaries. We choose here the approach developed in [19] and extend it to the mixture model detailed in $[21,22]$ to allow distinct phases or mixtures to enter and leave the domain.

After a presentation of the two-component model in the first section, with a focus on the turbulence and relative velocity closure, its SPH numerical implementation is detailed in a second part, giving careful attention to the open boundary formulation for which the theoretical developments can be found in appendices. This model is then applied to two two-dimensional air entrainment test cases: a stepped spillway for interfacial aeration and a plunging jet for local aeration. Finally, a three-dimensional industrial case is considered.

\section{Two-component model}

We consider in the following an air-water flow. We first detail the notations used in this work to model such a flow and then the equations solved.

\subsection{Notations}

We consider the volume ${ }^{1}$ presented in Figure 1a. The presence of air and water, denoted by $\alpha$ and $\beta$ respectively, is taken into account through volume fractions, as illustrated schematically in Figure 1b. Phase quantities listed in Table 1 allow us to compute mixture quantities detailed in Table 2. Among the different choices for mixture values, in order to ease the numerical treatment at high density ratios, we choose the volume-based formulation employing volume fraction ${ }^{2}$ for phase description and volumetric flux as mixture velocity. To preserve the symmetry of

\footnotetext{
1 The volume represents the SPH particle that will be introduced in the numerical section.

$2 \alpha$ and $\beta$ denote the volume fractions together with the phase names, without any risk of confusion.
} 


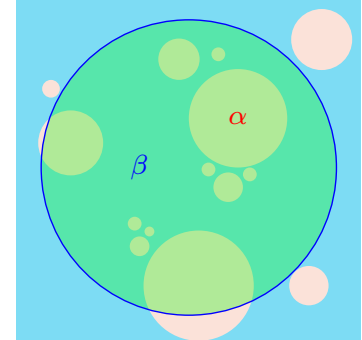

(a) Schematic view

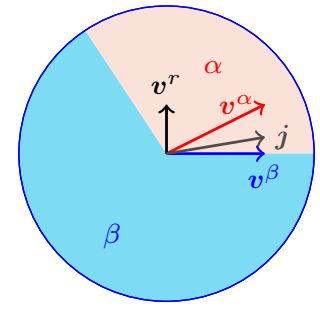

(b) Velocities

Fig. 1: Control volume in a two-component flow (gas phase in red, liquid phase in blue), the corresponding volume fractions and velocity fields [21,22].

the system, relative velocities are here preferred to drift/diffusion velocities. Let us underline that, by definition of the volume fractions, one has:

$$
\alpha+\beta=1
$$

Table 1: Phase quantities $(k=\alpha$ or $\beta)$

\begin{tabular}{l|l} 
Volume & $V^{k}$ \\
Mass & $m^{k}$ \\
Density & $\rho^{k}=m^{k} / V^{k}$ \\
Pressure & $p^{k}$
\end{tabular}

\begin{tabular}{l|c} 
Velocity & $\boldsymbol{v}^{k}$ \\
Dispersed phase diameter & $d^{k}$ \\
Kinematic viscosity & $\nu^{k}$ \\
Dynamic viscosity & $\mu^{k}$
\end{tabular}

Table 2: Mixture quantities

\begin{tabular}{l|l} 
Volume & $V=V^{\alpha}+V^{\beta}$ \\
Inverse volume & $\sigma=1 / V$ \\
Mass & $m=m^{\alpha}+m^{\beta}$ \\
Density & $\rho=m / V=\alpha \rho^{\alpha}+\beta \rho^{\beta}$ \\
Volume fractions & $\alpha=V^{\alpha} / V$ and $\beta=V^{\beta} / V$ \\
Mass fractions & $Y^{\alpha}=\alpha \rho^{\alpha} / \rho$ and $Y^{\beta}=\beta \rho^{\beta} / \rho$ \\
Mixture velocity & \\
w.r.t. the volume centre & $j=\alpha \boldsymbol{v}^{\alpha}+\beta \boldsymbol{v}^{\beta}$ \\
Mixture velocity & \\
w.r.t. the mass centre & $\boldsymbol{u}=Y^{\alpha} \boldsymbol{v}^{\alpha}+Y^{\beta} \boldsymbol{v}^{\beta}$ \\
Relative velocity & $\boldsymbol{v}^{r}=\boldsymbol{v}^{\alpha}-\boldsymbol{v}^{\beta}$ \\
Drift velocity & $\boldsymbol{v}^{\alpha}-\boldsymbol{j}$ \\
Diffusion velocity & $\boldsymbol{v}^{\alpha}-\boldsymbol{u}$ \\
Mixture pressure & $p$
\end{tabular}


2.2 Derivation of the continuous model

Following [31], the averaged model for two-phase flows gives the following set of continuous phase equations:

$$
\begin{gathered}
\frac{\partial \alpha \rho^{\alpha}}{\partial t}+\nabla \cdot\left(\alpha \rho^{\alpha} \boldsymbol{v}^{\alpha}\right)=0 \\
\frac{\partial \alpha \rho^{\alpha} \boldsymbol{v}^{\alpha}}{\partial t}+\nabla \cdot\left(\alpha \rho^{\alpha} \boldsymbol{v}^{\alpha} \otimes \boldsymbol{v}^{\alpha}\right) \\
=-\alpha \boldsymbol{\nabla} p^{\alpha}+\boldsymbol{\nabla} \cdot\left[\alpha\left(\boldsymbol{T}^{\alpha}+\boldsymbol{T}^{\alpha, T}\right)\right]+\alpha \rho^{\alpha} \boldsymbol{g}+\boldsymbol{M}^{\alpha}
\end{gathered}
$$

where $\boldsymbol{T}^{\alpha}=\mu^{\alpha}\left(\boldsymbol{\nabla} \boldsymbol{v}^{\alpha}+{ }^{t} \boldsymbol{\nabla} \boldsymbol{v}^{\alpha}\right)$ stands for the viscous stress tensor, $\boldsymbol{T}^{\alpha, T}$ encompasses turbulent effects, $\boldsymbol{g}$ is the gravity vector and $\boldsymbol{M}^{\alpha}$ the macroscopic interfacial momentum transfer. We assume in the following that there is no mass transfer nor surface tension effects ${ }^{3}$ and that there is a mechanical equilibrium of partial pressures $p=p^{\alpha}=p^{\beta}$ as pressure relaxation time is generally small compared to other characteristic times of the flow, as illustrated in [37] in case of bubbly flows. With this set of assumptions, one has $\boldsymbol{M}^{\beta}=-\boldsymbol{M}^{\alpha}$. Combining (2) and (3), one can write an evolution equation for the relative velocity. Under the assumptions of local equilibrium, and assuming that pressure, viscosity and momentum transfers are the leading terms, this relation leads to:

$$
\begin{aligned}
\boldsymbol{M}^{\alpha}= & \alpha \beta \frac{\rho^{\beta}-\rho^{\alpha}}{\rho} \nabla p \\
& -\frac{\beta \rho^{\beta}}{\rho} \nabla \cdot\left(\alpha \mu^{\alpha}\left(\nabla \boldsymbol{v}^{\alpha}+{ }^{t} \boldsymbol{\nabla} \boldsymbol{v}^{\alpha}\right)\right) \\
& +\frac{\alpha \rho^{\alpha}}{\rho} \nabla \cdot\left(\beta \mu^{\beta}\left(\nabla \boldsymbol{v}^{\beta}+{ }^{t} \boldsymbol{\nabla} \boldsymbol{v}^{\beta}\right)\right)
\end{aligned}
$$

In the following, we will work with the assumption of constant phase densities (i.e. $\rho^{\alpha}=$ cst and $\rho^{\beta}=$ cst).

\subsubsection{Total volume conservation}

Summing on both phases (2) divided by phase densities leads to a divergence-free mixture velocity field as we work under a constant phase density assumption. The weakly-compressible framework retained in the SPH approach will however make us relax this condition to solve the volume conservation equation (written in terms of inverse volume $\sigma=1 / V)$ :

$$
\frac{d \sigma}{d t}=-\sigma \nabla \cdot j
$$

3 In presence of surface tension, $\boldsymbol{M}^{\alpha}+\boldsymbol{M}^{\beta}=-2 H_{\beta \alpha} \sigma_{S}$ where $H_{\beta \alpha}$ is the mean curvature between phases $\alpha$ and $\beta$, and $\sigma_{S}$ is the surface tension coefficient (relation written considering that there are no extra interfacial momentum source accounting for local surface forces resulting from pressure and shear stress deviations from interfacial average values). 


\subsubsection{Volume fraction equation}

Dividing (2) by the phase density leads to the volume fraction equation:

$$
\frac{\partial \alpha}{\partial t}+\boldsymbol{j} \cdot \nabla \alpha=-\nabla \cdot\left(\alpha \beta \boldsymbol{v}^{r}\right)
$$

\subsubsection{Momentum equation}

Summing on both phases (3) divided by phase density and using the divergence free property of the mixture velocity field ${ }^{4}$ leads to:

$$
\begin{aligned}
& \frac{\partial \boldsymbol{j}}{\partial t}+\boldsymbol{j} \cdot \boldsymbol{\nabla} \boldsymbol{j}=-\frac{1}{\rho} \boldsymbol{\nabla} p+\boldsymbol{g}-\boldsymbol{\nabla}\left(\alpha \beta \boldsymbol{v}^{r} \otimes \boldsymbol{v}^{r}\right) \\
& +\frac{1}{\rho} \nabla \cdot\left[\rho\left(\nu+\nu_{T}\right)\left(\nabla j+{ }^{t} \nabla j\right)\right]
\end{aligned}
$$

where we have used (4) and assumed the turbulent effects could be modeled as a turbulent viscosity $\nu^{T}$ added to the viscosity $\nu=\left(\alpha \mu^{\alpha}+\beta \mu^{\beta}\right) / \rho$. Let us underline that, in this first approach, we have neglected a viscous term depending on relative velocities of the form $\frac{1}{\rho} \boldsymbol{\nabla} \cdot\left[\alpha\left(\mu^{\alpha}-\mu^{\beta}\right)\left(\boldsymbol{\nabla}\left(\beta \boldsymbol{v}^{r}\right)+{ }^{t} \boldsymbol{\nabla}\left(\beta \boldsymbol{v}^{r}\right)\right)\right]$ that includes an interfacial stress closure suggested by [31] assuming $\alpha$ is dispersed. The closure (4) allows one to recover the classical form of the pressure term for the averaged flow, and therefore restores the hydrostatic equilibrium when it occurs.

\subsection{Turbulence model}

Air entrainment and turbulence are intrinsically related as the inception of air bubble entrapment is generally related to the turbulent shear stresses overcoming the resisting forces of gravity and surface tension [10] as exemplified by the criterion of [28]. Turbulent effects shall therefore be included in the model. To this end, a $k-\epsilon$ approach is used in the standard way described in [39]. Several references in the literature $[42,45]$ dealing with air entrainment cases considered the realizable $k-\epsilon$ model developed in [54] in combination with the mixture model approach. [45] highlighted that among the $k-\epsilon$ models, the RNG approach performed better in the cases they considered. Even though numerical results might benefit from these modifications of the standard model (e.g. backward flows, rotations...), to limit additional modeling efforts (boundary terms, variable density effects), the standard approach with usual constant values given in Table 3 was used, applied to mixture quantities. The only modification is the introduction of the volume fraction of water as a weighting coefficient of the production term: for high-speed flows, the expected prominent turbulent contribution should come from water

\footnotetext{
4 The divergence-free property is relaxed for the continuity resolution but kept in the momentum equation resolution, as classically done in the weakly compressible SPH framework.
} 
Table 3: Constants of the $k-\epsilon$ model.

\begin{tabular}{|c|c|c|c|c|c|c|}
\hline$C_{\nu}$ & $C_{\mu}$ & $\sigma_{k}$ & $\sigma_{\epsilon}$ & $C_{\epsilon 1}$ & $C_{\epsilon 2}$ & $\kappa$ \\
\hline 5.2 & 0.09 & 1.0 & 1.3 & 1.44 & 1.92 & 0.41 \\
\hline
\end{tabular}

turbulence ${ }^{5}$.

$$
\begin{aligned}
& \frac{d k}{d t}=\frac{1}{\rho} \nabla \cdot\left[\left(\mu+\frac{\mu_{T}}{\sigma_{k}}\right) \nabla k\right]+\mathbb{P}-\epsilon \\
& \frac{d \epsilon}{d t}=\frac{1}{\rho} \nabla \cdot\left[\left(\mu+\frac{\mu_{T}}{\sigma_{\epsilon}}\right) \nabla \epsilon\right]+\frac{\epsilon}{k}\left(C_{\epsilon_{1}} \mathbb{P}-C_{\epsilon_{2}} \epsilon\right)
\end{aligned}
$$

where

$$
\begin{aligned}
\mathbb{P} & =\beta \min \left(\sqrt{C_{\mu}} k S, \nu_{T} S^{2}\right) \\
S & =\sqrt{2 s: s} \\
\boldsymbol{s} & =\frac{1}{2}\left(\boldsymbol{\nabla} \boldsymbol{j}+{ }^{t} \boldsymbol{\nabla} \boldsymbol{j}\right)
\end{aligned}
$$

The turbulent viscosity is computed as:

$$
\nu_{T}=C_{\mu} \frac{k^{2}}{\epsilon}
$$

Developing a mixture $k-\epsilon$ model adapted to the modeling choices made is part of the further work, with special care given to the inclusion of a buoyancy term. Due to the presence of walls denoted $\partial \Omega_{w}$, the turbulence becomes anisotropic and shearing effects resulting from the high velocity gradient to fit the wall no-slip condition increase the production of turbulence. The development of boundary layers is pivotal in many air entrainment industrial applications so that the near-wall turbulence must be properly modeled. To avoid the need of a refined discretization near the wall, semi-empirical relations can be used to introduce wall functions: in the Lagrangian framework, an Eulerian mean velocity with non-zero tangential value is set at the wall to get the adequate wall shear stress and used for the rateof-strain and viscous force computations. Let us introduce $y^{+}$the dimensionless distance to a wall defined as:

$$
y^{+}=\frac{y u_{\star}}{\nu}
$$

where $y$ is the wall normal coordinate, $\nu$ a mixture kinematic viscosity to be defined (as we considered the mixture as a whole, but this is also an approximation as the usual wall law considers a single fluid) and $u_{\star}$ the friction velocity defined as:

$$
u_{\star}^{2}=\left.\nu \frac{d j_{\tau}}{d y}\right|_{\partial \Omega_{w}}
$$

\footnotetext{
5 In this work we are mainly concerned by what happens in the water phase and mixture region. The modelling of the air phase appears as a requirement of the mixture model to exchange phases between particles when air entrainment occurs but we do not intend here to be precise in the resolution of the air phase dynamics, that prove to be affected by numerical instabilities in the SPH framework.
} 
with $j_{\tau}$ the wall tangential velocity. The shear stress at the wall follows:

$$
\mathbb{T}_{w}=-\rho u_{\star}^{2} \frac{\boldsymbol{j}}{|\boldsymbol{j}|}
$$

and the friction velocity is assumed to satisfy the logarithmic law for a smooth velocity profile:

$$
\frac{j_{\tau}}{u_{\star}}=\frac{1}{\kappa} \ln \left(y_{+}\right)+C_{\nu}
$$

where $\kappa$ is the Von Kármán constant. Following the work of [40], Neumann conditions derived from the equilibrium $\mathbb{P}=\epsilon$ in the logarithmic zone for fully developed turbulence are used in the second order differential operator computation close to the walls (small $y$ ):

$$
\left\{\begin{array}{l}
\left.\nabla k \cdot \boldsymbol{n}\right|_{\partial \Omega_{w}}=0 \\
\left.\nabla \epsilon \cdot \boldsymbol{n}\right|_{\partial \Omega_{w}}=-\frac{u_{\star}^{3}}{\kappa y^{2}}
\end{array}\right.
$$

At an imposed velocity boundary, the turbulent kinetic energy and its dissipation are imposed whereas at an imposed pressure boundary they are computed through an homogeneous Neumann condition.

\subsection{Relative velocity closure}

The two-component behavior of the flow is ruled by the relative velocity that needs to be closed by an algebraic relation in the mixture model. It is flow-dependent and therefore relies on some hypotheses about the regime considered.

Proposed model Phases can move at different velocities but it is assumed that they reach a local equilibrium over a short spatial length scale. A proposed model for this closure can be deduced by equalling ${ }^{6}$ the relation (4) with the following momentum transfer term:

$$
\boldsymbol{M}^{\alpha}=-\alpha \Gamma \boldsymbol{V}^{r}
$$

where $\boldsymbol{V}^{r}$ stands for the averaged value of the local relative velocity to distinguish from $\boldsymbol{v}^{r}$ that arises from the difference of averaged phase velocities: the difference between these relative velocities can be approximated by a mean drift velocity $\boldsymbol{V}^{d}$ related to the correlation between the instant distribution of the dispersed phase and the large scale turbulent fluid motion [55]. It is modeled here with a Standard Gradient Diffusion Hypothesis [47] and a diffusivity factor, ratio of the turbulent viscosity and the turbulent Schmidt number $\left(\mathrm{Sc}_{T}=1\right.$ here):

$$
\boldsymbol{V}^{d}=-\frac{\nu_{T}}{\mathrm{Sc}_{T}} \frac{\nabla \alpha}{\alpha \beta}
$$

The coefficient $\Gamma$ writes:

$$
\Gamma=\frac{3 C_{D} \operatorname{Re} \mu^{\beta}}{4\left(d^{\alpha}\right)^{2} \beta^{n-1}}
$$

\footnotetext{
${ }^{6}$ With the approximation of neglecting the viscous contribution.
} 
where $n$ is a power accounting for a hindering effect (i.e. the effect of the presence of other particles in the neighborhood distorting the flow field; we will take $n=1$ here), $d^{\alpha}$ is the dispersed phase size (assumption of monodisperse spherical bubbles or drops) and the drag coefficient $C_{D}$ is computed using an extension of the Schiller \& Naumann expression [53,43,14]:

$$
C_{D}= \begin{cases}\frac{24}{\operatorname{Re}}\left(1+0.15 \operatorname{Re}^{0.687}\right) & \text { if } \operatorname{Re} \leq 1000 \\ 0.44 & \text { if } \operatorname{Re}>1000\end{cases}
$$

where $\operatorname{Re}=\left|\boldsymbol{V}^{r}\right| d^{\alpha} / \nu^{\beta}$, appropriate for the bubble size considered in this work, around $1 \mathrm{~mm}^{7}$. The resulting relative velocity writes:

$$
\boldsymbol{v}^{r}=\boldsymbol{V}^{r}-\boldsymbol{V}^{d}=\frac{\beta\left(\rho^{\alpha}-\rho^{\beta}\right)}{\Gamma} \frac{\nabla p}{\rho}-\frac{\nu_{T}}{\mathrm{Sc}_{T}} \frac{\nabla \alpha}{\alpha \beta}
$$

that retains two main inter-phase forces, namely a drag term and a turbulent diffusion term. We have assumed in these expressions that $\alpha$ was the dispersed phase. An inherent limitation of this approach lies in the choice of $d^{\alpha}$. Solving an equation on the interfacial area density or Population Balance Equations could allow one to follow the size of the dispersed phase. In this first approach, we consider here a constant value of $d^{\alpha}$, given by the user. Experimental results can help us to chose an adequate value (e.g. experimental mean Sauter diameter).

Hydrostatic approximation Due to the very noisy nature of the pressure field in the SPH approach retained here, it did not appear numerically stable to use directly the pressure gradient in the relative velocity computation. An additional approximation was therefore made to use the hydrostatic value of the pressure gradient $\nabla p=\rho \boldsymbol{g}$ in this model. This choice decouples the phase evolution from the dynamics generated by the momentum equation. In absence of diffusion, the only possible transfers occur in the gravity direction. It does not allow one to reproduce effects generated by a non-hydrostatic pressure gradient as described by [33].

Explicit computation of the relative velocity Because of the presence of $\boldsymbol{V}^{r}$ in the Reynolds number, the relative velocity definition is implicit and it would require some iterations to converge. To save computational time, we make the following reasoning ${ }^{8}$. Noting $R_{\rho}=\left(\rho^{\alpha}-\rho^{\beta}\right) / \rho^{\beta}$, we first define the Reynolds number function $f_{R}$ :

$$
f_{R}(\operatorname{Re})=\operatorname{Re}\left(1+0.15(\operatorname{Re})^{0.687}\right)=\frac{\left(d^{\alpha}\right)^{3} \beta^{n}}{18\left(\nu^{\beta}\right)^{2}}\left|R_{\rho} \boldsymbol{g}\right|
$$

7 This choice is related to experimental measurements on the considered applications cases, [11] and [4], for which peaks of bubble chord distributions were noticed around 1-2 mm.

8 This approach proves to give correct values of the relative velocity without iteration : the error is below $1 \%$ for $R e>104$ and below $0.2 \%$ for $R e>218$. The peak of error is reached for very small Reynolds number, at $R e \sim 2$ where it reaches $10 \%$. However, in the practical air-water applications considered, we fall within the $1 \%$ error range. 
An approximation of $f_{R}^{-1}$ over $\operatorname{Re} \in[0 ; 1000]$ is:

$$
\operatorname{Re}=c_{R}\left(1-\frac{1}{1+\left(\frac{f_{R}(\operatorname{Re})}{b_{R}}\right)^{a_{R}}}\right)
$$

where the coefficients $a_{R}=0.6301226, b_{R}=2124552$ and $c_{R}=21037.87$ computed numerically. We propose the following scheme:

- Initialize $\operatorname{Re}_{0}=24 / \operatorname{Re}$

- Compute $f_{R}(\mathrm{Re})$ using (24)

- Deduce Re through (25)

- Then

- If $\operatorname{Re}<1000$, we compute a first guess as

$$
\boldsymbol{V}^{r}=\frac{4\left(d^{\alpha}\right)^{2} \beta^{n}}{3 \nu^{\beta} C_{\mathrm{D}}(\operatorname{Re}) \operatorname{Re}} R_{\rho} \boldsymbol{g}
$$

- Else $C_{D}(R e)=0.44$ and the relative velocity is written explicitly

$$
\boldsymbol{V}^{r}=\sqrt{\frac{4 d^{\alpha} \beta^{n}}{3 C_{\mathrm{D}} \rho^{\beta}\left|R_{\rho} \boldsymbol{g}\right|}} R_{\rho} \boldsymbol{g}
$$

If $\operatorname{Re}^{\alpha}<1000$, the first guess is already a good estimate generally. In the other case, the computation is explicit.

\subsection{State equation}

As detailed in $[21,22]$ for the SPH mixture model, within the Weakly Compressible SPH (WCSPH) framework, we link the pressure to a ratio of inverse volumes instead of densities:

$$
p=\left(\alpha \rho^{\alpha}\left(c^{\alpha}\right)^{2}+\beta \rho^{\beta}\left(c^{\beta}\right)^{2}\right)\left(\frac{\sigma}{\sigma_{0}}-1\right)+p_{B}
$$

We have now a complete set of equations (5), (6), (7), (8), (23) and (28) to solve numerically, complemented by the Lagrangian velocity used to move the SPH particles:

$$
\frac{d \boldsymbol{r}}{d t}=j
$$

This formulation reduces to a one-phase formulation for null or unit volume fraction. 


\section{SPH formulation}

We describe in this section the SPH formulation retained to solve discretely the preceding set of equations and make a special focus on the open boundary formulation. We adopt the convention that, for a field $A$ in a domain $\Omega$ of dimension $d$, the discretized SPH form after the interpolation process is $A_{b}=A\left(\mathbf{r}_{b}, t\right)$ where $\mathbf{r}_{b}$ is the particle position vector of the particle $b$ and $t$ is the time. The particle diameter is denoted $\delta r$. Numerical simulations were performed with an in-house code relying on the WCSPH Lagrangian approach and running on GPU. The general description of the SPH model can be found in [22]. We will stress here the additions and modifications performed to handle air-water flows.

\subsection{SPH formulation}

The continuous SPH interpolation is performed with the Wendland $C^{2}$ kernel [63] denoted $w$. For a pair of particles $(a, b), w_{a b}=w\left(\boldsymbol{r}_{a b}\right)$ where $\boldsymbol{r}_{a b}=\boldsymbol{r}_{a}-\boldsymbol{r}_{b}$ and the kernel gradient writes $\nabla w_{a b}=\nabla_{a} w\left(\boldsymbol{r}_{a}-\boldsymbol{r}_{b}\right)$. We work with the SPH differential operators adapted to a multifluid framework following [23] with the specific volume formulation originally introduced by [29]. Boundaries are treated with the Unified Semi-Analytical Wall (USAW) boundary conditions described in [20] and are able to deal with open boundaries [19]: boundaries $\partial \Omega$ of the domain consist of a set vertex particles $v \in \mathcal{V}$ connected by a set of segments $s \in \mathcal{S}$ of size $\delta r$ that forms a mesh of the boundary. $\mathcal{P}$ refers to the set of fluid particles $\mathcal{F} \cup \mathcal{V}$ : vertex particles are indeed truncated fluid particles with a volume computed with respect to a reference volume through a fraction $\theta$ defined as the angle between two connected segments divided by $2 \pi$ in $2 \mathrm{D}$ as shown on Figure $2 \mathrm{~b}$. $\theta$ equals $1 / 2$ for segments and 1 for fluid particles. As illustrated on Figure 2a, the kernel may intersect a boundary, giving rise to a boundary term in the SPH interpolation of the differential operators. This truncation of the kernel by a boundary introduces a renormalization factor $\gamma$ to the SPH interpolation as described by [20]:

$$
\gamma_{a}=\int_{\Omega_{r_{a}} \cap \Omega} w\left(\boldsymbol{r}_{a}-\boldsymbol{r}\right) d \boldsymbol{r}
$$

where $\Omega_{\boldsymbol{r}_{a}}$ denotes the kernel support of the particle $a$. Moreover, following [20], the contributions to the gradient of $\gamma$ that appear in the discrete differential operators are:

$$
\nabla \gamma_{a s}=\left(\int_{s} w(r) d S\right) \boldsymbol{n}_{s}
$$

where $\boldsymbol{n}_{s}$ is the inward unit normal to the boundary segment $s$. These values can be computed analytically as explained in [20].

\subsection{Phase and mixture volumes}

The resolution of the total volume equation (5) is addressed in Appendix A while the resolution of the phase volume equation (6) was thoroughly detailed in [21,22]. However the special choice of relative velocity requires to make an adaptation of 


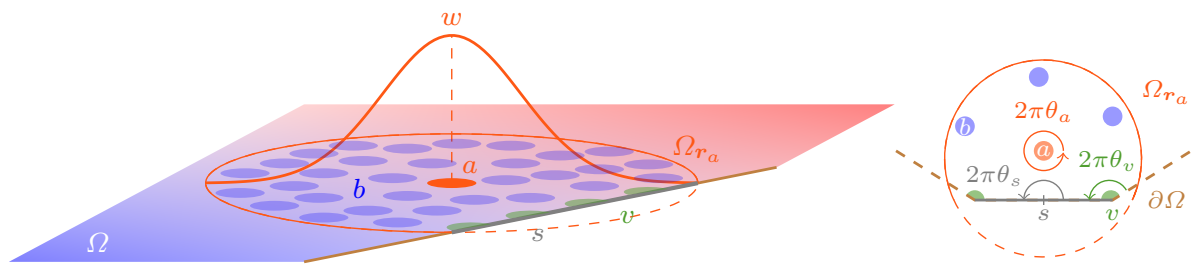

(a) SPH interpolation kernel.

(b) $\mathrm{SPH}$ interpolation kernel.

Fig. 2: Description of the SPH particles.

the diffusion term compared to [21,22]: the eddy viscosity indeed varies and $\nabla \alpha$ is divided by $\alpha \beta$ so that the discrete phase volume equation now writes:

$$
\begin{aligned}
& \frac{d V_{a}^{\alpha}}{d t}=\alpha_{a} \frac{d V_{a}}{d t}-2 \frac{V_{a}}{\gamma_{a}} \sum_{b \in \mathcal{F}} V_{b}\left(\alpha_{a} \beta_{b}\left[\boldsymbol{V}_{a \mid b}^{r} \cdot \nabla w_{a b}\right]^{+}+\alpha_{b} \beta_{a}\left[\boldsymbol{V}_{a \mid b}^{r} \cdot \nabla w_{a b}\right]^{-}\right) \\
& +\frac{V_{a}}{\gamma_{a}} \sum_{b \in \mathcal{F}} V_{b} \frac{\nu_{T, a}+\nu_{T, b}}{\mathrm{Sc}_{T}} \frac{\alpha_{a}-\alpha_{b}}{r_{a b}} \frac{\boldsymbol{r}_{a b} \cdot \nabla w_{a b}}{r_{a b}}
\end{aligned}
$$

where $\mathcal{F}$ refers to the set of free fluid particles as no boundary term is included in this operator, $\boldsymbol{V}_{a \mid b}^{r}=\left(\boldsymbol{V}_{a}^{r}+\boldsymbol{V}_{b}^{r}\right) / 2$. The condition for positiveness is also changed with a different relative velocity closure to:

$$
\mathrm{C}_{\alpha}=\frac{v_{0 \max } \delta t}{\sigma_{e}} \leq \xi_{\alpha}\left(\eta_{\alpha}+\frac{1}{\mathrm{Pe}_{\sigma}}\right)^{-1}
$$

where the numerical Péclet number writes:

$$
\mathrm{Pe}_{\sigma}=\frac{v_{0 \max } \sigma_{e} \mathrm{Sc}_{T}}{\nu_{T, \max }}
$$

with $\sigma_{e}$ the standard kernel deviation, $v_{0 \text { max }}=\max _{a \in \mathcal{F}}\left(\left|\boldsymbol{V}_{a}^{r}\right|\right), \nu_{T, \max }=\max _{a \in \mathcal{F}}\left(\nu_{T, a}\right)$ and for which we used the same values of $\left(\xi_{\alpha}, \eta_{\alpha}\right)$ as in [22] due to the proximity of the formulations. Let us underline that in the turbulent flows considered in the following and consistently with the assumptions of the mixture model, the norm of the relative velocity is small compared to the flow velocity so that this condition will not be the strongest constraint on the time step. As detailed in [21,22], volume diffusion was introduced when phase volumes are updated to prevent the development of instabilities linked to the colocated nature of SPH.

$$
V_{a}^{\alpha, n+1}=V_{a}^{\alpha, \star}+\frac{\delta t}{\gamma_{a}} \Lambda \alpha_{a}^{n} \mathcal{D}_{a}^{n}
$$

where $V_{a}^{\alpha, \star}$ is the phase volume before its modification by the diffusion term that writes:

$$
\mathcal{D}_{a}^{n}=2 V_{a} \delta t \sum_{b \in \mathcal{F}} V_{b} \frac{1}{r_{a b}}\left(\frac{2}{\rho_{a}+\rho_{b}}\left(p_{a}-p_{b}\right)-\boldsymbol{g} \cdot \boldsymbol{r}_{a b}\right) \nabla w_{a b}
$$

The weighting coefficient $\Lambda$ of this volume diffusion is equal to 0.1 unless otherwise specified. 
3.3 Focus on the momentum equation

The discrete counterpart of the momentum equation (3) writes:

$$
\frac{d \boldsymbol{j}_{a}}{d t}=-\frac{1}{\rho_{a}} \boldsymbol{G}_{a}^{+}\left\{p_{b}\right\}+\frac{1}{\rho_{a}} \boldsymbol{L}_{a}\left\{\nu_{b}, \boldsymbol{j}_{b}\right\}+\boldsymbol{g}-\boldsymbol{D}_{a}\left\{\alpha_{b} \beta_{b} \boldsymbol{v}_{b}^{r} \otimes \boldsymbol{v}_{b}^{r}\right\}
$$

the operators being specified in the following sections.

\subsubsection{Pressure gradient}

Spurious behaviors near the interface between air and water exist and originates from the SPH continuous interpolation process. For a given particle $a$ in the $\alpha$ phase whose kernel support $\Omega_{\boldsymbol{r}_{a}}$ is shared between $\Omega^{\alpha}$ (phase $\alpha$ ) and $\Omega^{\beta}$ (phase $\beta$ ), the continuous SPH interpolation for the pressure gradient writes:

$$
\left[\frac{\nabla p}{\rho}\right](\boldsymbol{r})=\frac{1}{\rho(\boldsymbol{r})} \int_{\Omega_{\boldsymbol{r}_{a}}} \boldsymbol{\nabla}_{\boldsymbol{r}} p\left(\boldsymbol{r}^{\prime}\right) w\left(\boldsymbol{r}-\boldsymbol{r}^{\prime}\right) d V^{\prime}
$$

After splitting between the phases and assuming an incompressible hydrostatic configuration, one gets:

$$
\left[\frac{\nabla p}{\rho}\right](\boldsymbol{r})=\left[1+\left(\frac{\rho^{\beta}}{\rho^{\alpha}}-1\right) \int_{\Omega_{r_{a}} \cap \Omega^{\beta}} w\left(\boldsymbol{r}-\boldsymbol{r}^{\prime}\right) d V^{\prime}\right] \boldsymbol{g}
$$

One can see that when $\rho^{\beta} \gg \rho^{\alpha}$, a spurious interface force appears, very large in the air phase, small in the water phase (exchange the superscripts $\alpha$ and $\beta$ ), and leads to a gap configuration as an equilibrium position, the air particles reducing the overlapping of the kernel support with the other phase. As far as the pressure gradient is concerned, the multiple tests made with different formulations suggested in the literature $[29,30,35]$, did not allow us to identify a completely satisfactory discrete expression: the multifluid operator of [23] together with a small background pressure (that shall remain limited to avoid to decrease the precision) was finally considered:

$\boldsymbol{G}_{a}^{+}\left\{A_{b}\right\}=\frac{1}{\gamma_{a} V_{a}} \sum_{b \in \mathcal{P}}\left(V_{a}^{2} p_{a}+V_{b}^{2} A_{b}\right) \nabla w_{a b}-\frac{1}{\gamma_{a} V_{a}} \sum_{s \in \mathcal{S}} \frac{1}{V_{s}}\left(V_{a}^{2} A_{a}+V_{s}^{2} A_{s}\right) \nabla \gamma_{a s}$

\subsubsection{Viscous stresses}

In the framework of the mixture model, the viscosity $\nu$ varies in space due to its dependence on the volume fraction. We used in the second operator of [44]:

$L_{a}\left\{B_{b}, A_{b}\right\}=\frac{2}{\gamma_{a}} \sum_{b \in \mathcal{P}} V_{b} \bar{B}_{a b} \frac{A_{a}-A_{b}}{r_{a b}^{2}} \boldsymbol{r}_{a b} \cdot \nabla w_{a b}-\frac{1}{\gamma_{a}} \sum_{s \in \mathcal{S}}\left(B_{a} \nabla A_{a}+B_{s} \boldsymbol{\nabla} A_{s}\right) \cdot \boldsymbol{\nabla} \gamma_{a s}$

with $\bar{B}_{a b}$ the harmonic mean of $B_{a}$ and $B_{b}$. However, while it allows one to take variable viscosity effects into account, it misses the transpose part of the viscous stresses. [18] and [61] detailed an operator taking this part into account. This is left for further work. For turbulence modeling, the standard $k-\epsilon$ model [47], adapted to the USAW framework in [41], is used. 


\subsubsection{Convective transfers}

With respect to the single-phase formulation, one can note two main differences with the mixture model: mixture quantities are used for the variables and an additional term linked to the relative velocity appears in (3) as particles are moved with the mixture velocity. In view of momentum conservation, an antisymmetric formulation is retained without boundary term:

$$
\boldsymbol{D}_{a}\left\{\alpha_{b} \beta_{b} \boldsymbol{v}_{b}^{r} \otimes \boldsymbol{v}_{b}^{r}\right\}=\frac{1}{\gamma_{a} V_{a}} \sum_{b \in \mathcal{F}}\left[V_{a}^{2} \alpha_{a} \beta_{a} \boldsymbol{v}_{a}^{r}\left(\boldsymbol{v}_{a}^{r} \cdot \nabla w_{a b}\right)+V_{b}^{2} \alpha_{b} \beta_{b} \boldsymbol{v}_{b}^{r}\left(\boldsymbol{v}_{b}^{r} \cdot \nabla w_{a b}\right)\right]
$$

This term proved to generate spurious behaviors along the air-water interface, generating artificial mixing, so that, if not specified, it was neglected in the application cases detailed hereafter. Similarly to the treatment of the term $\boldsymbol{\nabla} \cdot\left(\alpha \beta \boldsymbol{v}^{r}\right)$ in the phase volume equation (32), one could consider an upwind formulation of this convective transfer term: this is left for future investigations.

\subsection{Time marching scheme}

The detailed resolution of our system is as follows (superscript stars refer to intermediate values):

1. Relative velocity update of the drag part as in Section 2.4

2. Operator splitting for the momentum equation: velocity update

(a) Potential force step

$$
\boldsymbol{j}_{a}^{\star}=\boldsymbol{j}_{a}^{n}+\delta t\left(-\frac{1}{\rho_{a}^{n}} \boldsymbol{G}_{a}^{+}\left\{p_{b}^{n}\right\}+\boldsymbol{g}\right)
$$

(b) (Convective transfer step)

$$
\boldsymbol{j}_{a}^{\star \star}=\boldsymbol{j}_{a}^{\star}-\delta t \boldsymbol{D}_{a}\left\{\alpha_{b}^{n} \beta_{b}^{n} \boldsymbol{v}_{b}^{r, n} \otimes \boldsymbol{v}_{b}^{r, n}\right\}
$$

(c) $(k-\epsilon$ update to compute the turbulent viscosity as in Section 2.3)

$$
\begin{aligned}
k_{a}^{n+1} & =k_{a}^{n}+\delta t\left[\mathbb{P}_{a}^{n}-\epsilon_{a}^{n} k_{a}^{n+1} / k_{a}^{n}+L_{a}\left\{\mu_{b}^{n}+\frac{\mu_{b, T}^{n}}{\sigma_{k}}, k_{b}^{n}\right\} / \rho_{a}^{n}\right] \\
\epsilon_{a}^{n+1} & =\epsilon_{a}^{n}+\delta t\left[\epsilon_{a}^{n} / k_{a}^{n}\left(C_{\epsilon_{1}} \mathbb{P}_{a}^{n}-C_{\epsilon_{2}} \epsilon_{a}^{n}\right)+L_{a}\left\{\nu_{b}^{n}+\mu_{b, T}^{n} / \sigma_{\epsilon}, \epsilon_{b}^{n}\right\} / \rho_{a}^{n}\right] \\
\mathbb{P}_{a}^{n} & =\min \left(\sqrt{C_{\mu}} k_{a}^{n} S_{a}^{n}, \nu_{T, a}^{n}\left(S_{a}^{n}\right)^{2}\right) \\
\mathbb{S}_{a}^{n} & =\sqrt{2 \boldsymbol{S}_{a}^{n}: \boldsymbol{S}_{a}^{n}} \quad \text { with } \quad \boldsymbol{S}_{a}^{n}=\left(\boldsymbol{G}_{a}^{-}\left\{\boldsymbol{j}_{b}^{n}\right\}+{ }^{t} \boldsymbol{G}_{a}^{-}\left\{\boldsymbol{j}_{b}^{n}\right\}\right) / 2
\end{aligned}
$$

where the symmetric gradient operator is defined as:

$$
\boldsymbol{G}_{a}^{-}\left\{\boldsymbol{A}_{b}\right\}=\frac{V_{a}}{\gamma_{a}} \sum_{b \in \mathcal{P}}\left(A_{a}-A_{b}\right) \nabla w_{a b}-\frac{V_{a}}{\gamma_{a}} \sum_{s \in \mathcal{S}} \frac{1}{V_{s}}\left(A_{a}-A_{s}\right) \nabla \gamma_{a s}
$$

(d) Viscous force step

$$
\boldsymbol{j}_{a}^{n+1}=\boldsymbol{j}_{a}^{\star \star}+\frac{\delta t}{\rho_{a}^{n}} \boldsymbol{L}_{a}\left\{\mu_{b}^{n}+\mu_{T, b}^{n}, \boldsymbol{j}_{b}^{\star \star}\right\}
$$


3. Particle position update

$$
\boldsymbol{r}_{a}^{n+1}=\boldsymbol{r}_{a}^{n}+\delta t \boldsymbol{j}_{a}^{n+1}
$$

4. Total volume update with (64)

5. Phase volume computations with (32)

6. Computation of other flow features [22]

$$
m_{a}^{n+1}=\left(\alpha_{a}^{n+1} \rho^{\alpha}+\beta_{a}^{n+1} \rho^{\beta}\right) V_{0} \quad \rho_{a}^{n+1}=\frac{m_{a}^{n+1}}{V_{a}^{n+1}} \quad \alpha_{a}^{n+1}=\frac{V_{a}^{\alpha, n+1}}{V_{a}^{n+1}}
$$

where $V_{0}$ is a reference volume is defined as $V_{0}=\delta r^{d}$.

7. Pressure computation using state equation (28)

To comply with the saturation condition (1), we deduce $\beta=1-\alpha$ when needed in the implementation.

\subsection{Numerical stability}

The maximum step size for numerical stability, studied in detail in [62], is constrained by several numbers:

- The Courant-Friedrichs-Levy (CFL) defined as:

$$
\mathrm{C}_{\mathrm{CFL}}=\frac{c_{0} \delta t}{\sigma_{e}}
$$

- The viscous limitation:

$$
\mathrm{C}_{\mathrm{visc}}=\frac{\nu \delta t}{\sigma_{e}^{2}}
$$

- According to [20], the governing equation on $\gamma$ leads to the following condition:

$$
\mathrm{C}_{\gamma}=\delta t \max _{a \in \mathcal{P}}\left(\left|\nabla \gamma_{a s} \cdot \boldsymbol{v}_{a s}\right|\right)
$$

Hence, numerical stability is ensured for:

$$
\delta t \leq \min \left(\mathrm{C}_{\mathrm{CFL}} \frac{\sigma_{e}}{c_{0}}, \mathrm{C}_{\mathrm{visc}} \frac{\sigma_{e}^{2}}{\nu}, \mathrm{C}_{\gamma} \frac{1}{\max _{a \in \mathcal{P}}\left(\left|\nabla \gamma_{a s} \cdot \boldsymbol{v}_{a s}\right|\right)}\right)
$$

One has typically $\left(\mathrm{C}_{\mathrm{CFL}}, \mathrm{C}_{\mathrm{visc}}, \mathrm{C}_{\gamma}\right)=(0.76,0.45,0.004)$ in $2 \mathrm{D}$ and $(0.76,0.45,0.001)$ in $3 \mathrm{D}$. These conditions are complemented by the condition for positiveness of phase volumes (33). The convective transfer term (42) comprises an advective and a diffusion components and therefore requires a condition too. Nevertheless, as it was not included in most of the applications further detailed, we have not made the appropriate derivation. As long as the relative velocity stays small compared to the maximum velocity of the flow and the diffusion coefficient remains of the order of the (possibly turbulent) viscosity of the fluid, it seems reasonable to consider that this term will not introduce an additional stability condition. 
3.6 Precaution at initialization and at open boundaries

At initialization and at open boundaries, the interface is diffused over a few particles using a hyperbolic tangent profile for the volume fraction (going from 0 to 1): this prevents the initial rearrangement of the particles, perturbed by the high density ratio, from a crash of the simulations. The phases of particles of the bulk of the fluid are then separated progressively. One layer of particles of intermediate volume fraction can persist as the discretization may not allow phases to be perfectly separate with the available volumes.

\subsection{Required modeling of the lighter phase}

Modeling explicitly the light phase in this framework, at least with several particle layers so that kernels of the heavy phase are not truncated in case of free surface flows, appeared to be necessary to preserve the quantities of each phase, so that particles can effectively exchange volumes of each phase. In the case of air-water mixtures, for example, this means that a sufficiently thick air layer should be initially set above the water surface. Computations are therefore more expensive.

\subsection{Open boundaries}

The discretization of boundary conditions remains a main challenge for the SPH community, especially the open boundaries often required in practical engineering applications and for coupling with other numerical models. The most common approach consists in introducing buffer layers and has been addressed with variations by several authors $[2,57]$, sometimes using Riemann invariants $[38,59]$. Few articles displayed multifluid open boundaries: [25] following [46] introduced oil bubbles at the inlet of a water tank, [36] wrote an algorithm for the Incompressible SPH approach with mirror particles able to handle back-flowing water at an air inlet. [17] adapted the idea of [57] to handle multiphase flows. The approach retained here follows [19] and its generalization of USAW conditions with the resolution of a 1D Riemann problem at open boundaries. Though time consuming, it allows one to impose precisely the desired velocity or pressure profiles and deduce the missing quantities through a rigorous framework inspired from finite volume developments. The extension to the mixture model detailed here requires to take into account the additional volume fraction equation and the possible presence of mixtures at open boundaries. In the derivation, we will consider that there is no relative velocity at the boundary: the resolution of the consequent homogeneous system, where the volume (or mass) fraction is a transported quantity, was already studied in the finite volume approach, see for example [6]. We work here in a simplified framework of barotropic and subsonic flow.

Correction of the single-fluid formulation In Appendix B, a correction of the formalism was made compared to the method presented in [19]: for a shock wave, the relations were indeed derived as if the shock wave velocity was null. We derive here the relations without any approximation on the shock speed. Moreover it was considered that the equality of left and right states of the tangential velocity was an 
assumption. It is shown here that it is a consequence of the Riemann formulation. The test cases developed in [19] were run with these improvements and compared to the original formulation, namely the non-orthogonal flux on inlet/outlet in a square, a rapidly expanding pipe, $2 \mathrm{D}$ periodic free-surface water wave and a $2 \mathrm{D}$ solitary wave. Similar behaviors as in [19] were obtained: the computed solutions of the Riemann problems at boundaries in case of shock wave (the only different configuration) are close. Nevertheless, it is an improvement as seen on the 2D periodic free-surface water wave test case for which we get more regular fields near the open boundaries.

\subsubsection{Time integration of the continuity equation}

The continuity equation (5) resolution is modified as detailed in Appendix A to avoid spurious density variations close to the open boundaries.

\subsubsection{Update of vertex particles features}

Vertices have variable masses and volumes. Masses are computed similarly to [19] except that the mass flux on a vertex $v$ surrounded by segments $s$ is assessed using:

$$
\dot{m}_{v}=\frac{1}{2} \sum_{s \in \int} \frac{\alpha_{v} \rho^{\alpha}+\beta_{v} \rho^{\beta}}{\alpha_{s} \rho^{\alpha}+\beta_{s} \rho^{\beta}} \rho_{s} S_{s}\left(\boldsymbol{v}_{s}-\dot{j}_{s}\right) \cdot \boldsymbol{n}_{s}
$$

where $S_{s}$ is the measure of the boundary element $s$ and the factor $\left(\alpha_{v} \rho^{\alpha}+\beta_{v} \rho^{\beta}\right) /\left(\alpha_{s} \rho^{\alpha}+\beta_{s} \rho^{\beta}\right)$ has been added in the sum to prevent one fluid to disrupt the mass flux computation of the other near the interface.

\subsubsection{Pressure and velocity at open boundaries}

A 1D Riemann problem is solved at open boundaries to compute compatible pressure and velocity fields (i.e. deduce a velocity field from an imposed pressure profile, or conversely). Characteristic waves allow one to make the link between the exterior state (the boundary condition we impose) and the interior state (interpolated from the fluid values) and finally deduce the state at the boundary. Notations and derivations are presented in Appendix B. The relation between pressure and velocity fields will depend on the type of discontinuity associated to the eigenvalue $\lambda_{+1}$.

\section{Academic test cases}

The two-phase mixture SPH model was validated on 2D academic cases in [22], checking the conservation, realizability and reduction to single fluid formulation properties. We investigate here air-water mixtures with open boundaries. 


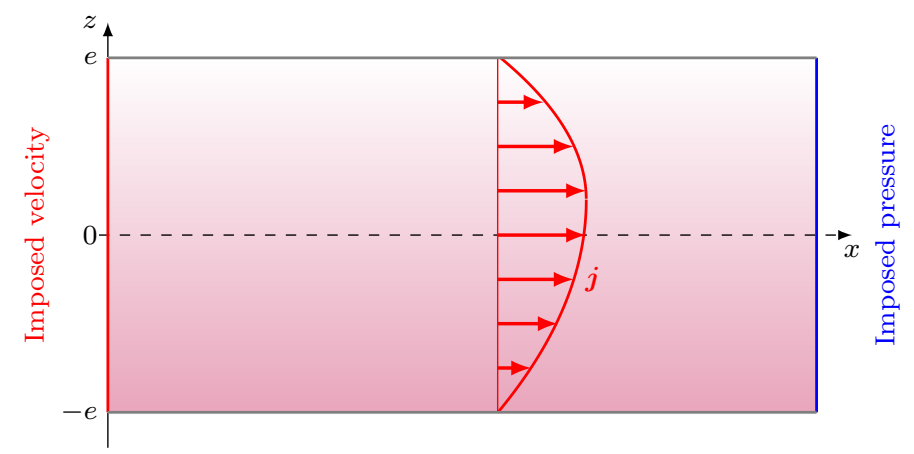

Fig. 3: Two-phase mixture Poiseuille flow with open boundaries: geometry.

4.1 Two-phase mixture Poiseuille flow

A two-phase mixture Poiseuille flow for which an analytical solution has been derived at steady state in Appendix $\mathrm{C}$ is employed to study the accuracy of the implementation of the model together with the correct implementation of the open boundaries. $\beta$ is approximated by 1 in the relative velocity closure and the diffusivity coefficient $\nu_{T} / \mathrm{Sc}_{T}$ is taken to a constant value $K^{9}$.

Description The geometry is described in Figure 3. The physical and numerical parameters are listed in Table 4. Following the solution described in Appendix C for a periodic case with an applied longitudinal force $F$, a two-phase mixture is imposed with the analytical profile at the inlet while the background pressure is imposed at the outlet. The volume fraction is imposed at both open boundaries and the variables are initialized with the analytical solution within the domain. There is no body force, but the gravity is used to compute the relative velocity.

Table 4: Parameters for the two-phase mixture Poiseuille flow with inlet/outlet.

\begin{tabular}{|c|rl|}
\hline$L$ & 10 & $\mathrm{~m}$ \\
\hline$\rho^{\alpha}$ & 1.23 & $\mathrm{~kg} / \mathrm{m}^{3}$ \\
\hline$\nu^{\alpha}$ & 0.03 & $\mathrm{~m}^{2} / \mathrm{s}$ \\
\hline$c^{\alpha}$ & 3.3 & $\mathrm{~m} / \mathrm{s}$ \\
\hline$d^{\alpha}$ & 0.2 & $\mathrm{~m}$ \\
\hline$\delta r$ & 0.01 & $\mathrm{~mm}$ \\
\hline
\end{tabular}

\begin{tabular}{|c|rl|}
\hline$e$ & 1 & $\mathrm{~m}$ \\
\hline$\rho^{\beta}$ & 1000 & $\mathrm{~kg} / \mathrm{m}^{3}$ \\
\hline$\nu^{\beta}$ & 0.03 & $\mathrm{~m}^{2} / \mathrm{s}$ \\
\hline$c^{\beta}$ & 3.3 & $\mathrm{~m} / \mathrm{s}$ \\
\hline$K$ & 0.1 & $\mathrm{~m}^{2} / \mathrm{s}$ \\
\hline$p_{B}$ & 1 & $\mathrm{~Pa}$ \\
\hline
\end{tabular}

Results Figure 4 highlights a smooth velocity field that is not altered near the open boundaries. One can check on Figures $5 \mathrm{a}$ and $5 \mathrm{~b}$ that the volume fraction

\footnotetext{
9 This test case aims at checking the correct implementation of the model, so that we chose a simplified formulation of the relative velocity to be able to derive an analytical formulation. These assumptions allow one to make the link between the general framework presented and this special verification test case.
} 


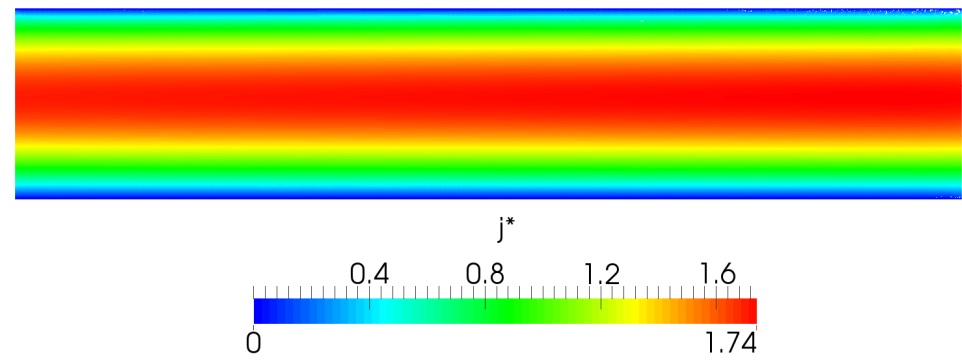

Fig. 4: Two-phase mixture Poiseuille flow with open boundaries: longitudinal velocity field at $t=50 \mathrm{~s}$.

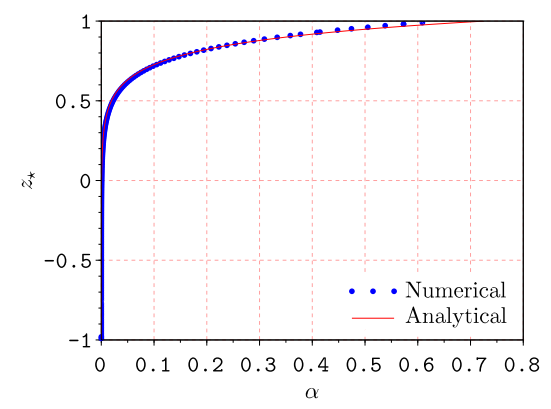

(a) Inlet.

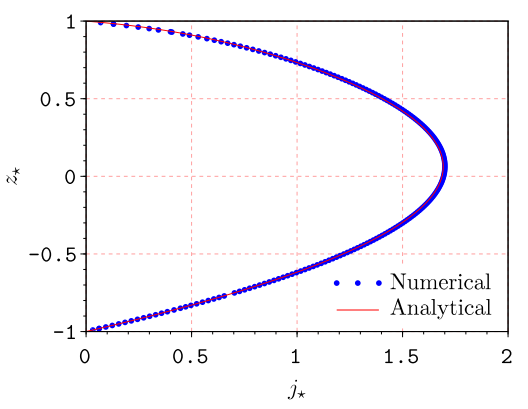

(b) Outlet.

Fig. 5: Two-phase mixture Poiseuille flow with open boundaries: comparison of the analytical and numerical profiles at $t=50 \mathrm{~s}$ at a section in the middle of the domain.

and velocity profiles fit well with the analytical solutions. The order of convergence was found to be of $1 / 2$ for the volume fraction and 1 for the longitudinal velocity on the periodic version of this test case in [22].

\subsection{An interfacial air entrainment test case: the stepped spillway}

Spillways form a classical structure of hydraulic works and provide a controlled release of flows from dams. Several geometries can be considered from small-slope to steep chutes, from smooth to step-pool spillways. Self-aeration is a common process in such structures and leads to several effects, namely flow bulking, drag reduction, cavitation mitigation and air-water mass transfers [10]. In the following, we will consider the skimming flow regime over a stepped spillway: a coherent water stream flows over a pseudo-bottom formed by the edges of step with in-between maintained recirculating vortices submitted to the shear forces of the main flow [10]. As described in [10], in this regime, a turbulent boundary layer develops from 

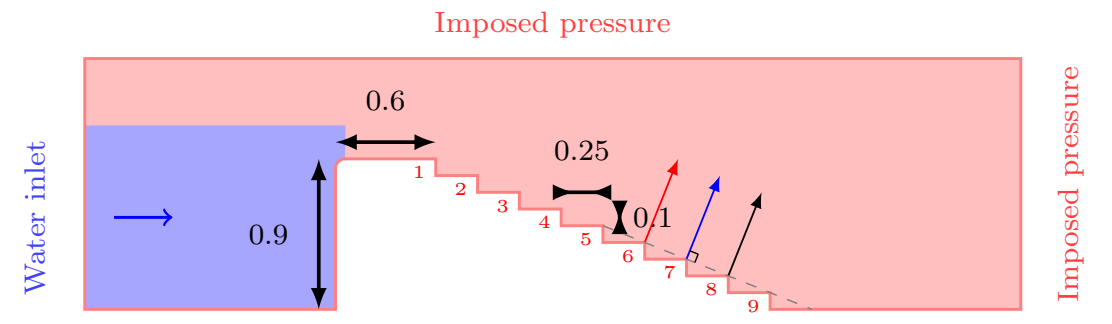

Fig. 6: Stepped spillway: geometry

the first steps. When it reaches the free surface, it triggers some entrainment and two distinct regions appear:

- A bubbly flow for small air volume fractions $(\alpha<0.3 / 0.4)$ formed of single and clusters of irregular air bubbles and air packets;

- A highly aerated flow for intermediate air volume fractions $(0.3 / 0.4<\alpha<0.9)$ formed by foam and air-water projections.

In such flows, the free surface is defined as the iso-air concentration line $\alpha=0.9$. According to [10], below the height $Y_{90}$ at which $\alpha=0.9$, the air-water flow behaves as an homogeneous mixture. The other characteristic features of such a flows for an in-going water volume flow rate $q^{\beta}$ per unit width are:

- Characteristic velocity $V_{90}$ for $y=Y_{90}$

- Mean air volume fraction $C_{\text {mean }}=\frac{1}{Y_{90}} \int_{0}^{Y_{90}} \alpha d y$

- Mean velocity $U_{W}=q^{\beta} /\left(Y_{90}\left(1-C_{\text {mean }}\right)\right)$

- Critical depth for a rectangular channel $d_{c}=\left[\left(q^{\beta}\right)^{2} / g\right]^{\frac{1}{3}}$

- Critical velocity for a rectangular channel $V_{c}=q^{\beta} / d_{c}$

Description The geometry of the model follows the experimental structure of [13] and is described in Figure 6. The slope is equal to $\theta_{s}=21.8^{\circ}$. The physical and numerical parameters are detailed in Table 5 . The $k-\epsilon$ model is employed for turbulence. Open boundaries conditions are used: water velocity is imposed on the right through a smooth logarithmic velocity profile with varying height according to the desired volume flow rate $^{10}$ :

$$
j_{x}=u_{\star}\left[\frac{1}{\kappa} \log \left(\frac{z u_{\star}}{\nu^{\beta}}\right)+C_{\nu}\right]
$$

with $z$ the vertical position and $u_{\star}$ the friction velocity deduced from iterations on the relation:

$$
u_{\star}=\frac{\kappa q^{\beta} / h_{u p}}{\log \left[\exp \left(\kappa C_{\nu}-1\right) h_{u p} u_{\star} / \nu^{\beta}\right]}
$$

\footnotetext{
10 This velocity profile is an arbitrary choice to impose the desired volume flow rate as the "true" profile is not known in this region near the structure. It is applied across the water height from the first layer of particles near the bottom to the free surface.
} 
with $h_{u p}$ the upstream water level left to vary and computed on the fly. This velocity is multiplied by $\min (1, t)$ to have an initial ramp to reach the desired volume flow rate without destabilizing strongly the domain during the first iterations. The background pressure is imposed on the upper boundary while at the outlet boundary we impose the hydrostatic pressure of the incoming mixture following:

$$
p(z)=p_{B}-\int_{z}^{H_{d}} \rho(z) g d z
$$

where the mixture density $\rho(z)$ is computed thanks to a SPH interpolation on the neighbors of the boundary particle and where at $z=H_{d}$ the top height of the outlet, one has $p\left(H_{d}\right)=p_{B}$. This condition is only approximately enforced: to avoid too heavy computations and due to the complexity of identifying in this Lagrangian framework which particles form the column above a given boundary particle, an additional table was introduced storing the value of the integral of (50) for all the column of particles above the particle of interest. It is initialized with the value for the hydrostatic pressure of an air column. It needs some iterations to adapt if abrupt changes occur. No turbulence is introduced at inlet. A volume diffusion coefficient $\Lambda=0.3$ was used to smooth the pressure field (instabilities developed at the interface for smaller values). The initial block of water reaches the

Table 5: Parameters for the stepped spillway case.

\begin{tabular}{|c|c|c|}
\hline$\rho^{\alpha}$ & 1.23 & $\mathrm{~kg} / \mathrm{m}^{3}$ \\
\hline$\nu^{\alpha}$ & $1.5610^{-5}$ & $\mathrm{~m}^{2} / \mathrm{s}$ \\
\hline$c^{\alpha}$ & 45 & $\mathrm{~m} / \mathrm{s}$ \\
\hline$d^{\alpha}$ & 1 & $\mathrm{~mm}$ \\
\hline$\delta r$ & 5 & $\mathrm{~mm}$ \\
\hline
\end{tabular}

\begin{tabular}{|c|rl|}
\hline$\rho^{\beta}$ & 1000 & $\mathrm{~kg} / \mathrm{m}^{3}$ \\
\hline$\nu^{\beta}$ & $10^{-6}$ & $\mathrm{~m}^{2} / \mathrm{s}$ \\
\hline$c^{\beta}$ & 45 & $\mathrm{~m} / \mathrm{s}$ \\
\hline$d^{\beta}$ & 1 & $\mathrm{~mm}$ \\
\hline$p_{B}$ & 500 & $\mathrm{~Pa}$ \\
\hline
\end{tabular}

level $h_{s}=1.1 \mathrm{~m}$ and falls down the steps before reaching the outlet boundary as a mixture. In our formulation, the transient of the flow going down the steps might not be accurate due to the assumptions of the formulation. We are interested in the steady state obtained after a few seconds of physical time when the volume fraction and velocity profiles does not vary significantly anymore. We considered different volume flow rates corresponding to different regimes tested by [13]: it is expected that the model performs better in the skimming regime where the variations of topology of the flow are confined to a smaller region.

Results Computations were performed with 400000 particles during around 14 hours of computational time on one graphic card GeForce GTX Titan Black for 10 s of physical time ${ }^{11}$. Profiles will be considered along the direction perpendicular to the slope (coordinate named $y$ ), starting from the edge of each step and are compared to measurements of [13]. The velocity component of interest is in the direction parallel to the slope. To post-process the profiles along the $y$ direction, a set of points spaced by $\delta r$ were introduced along the normal to the slope starting at the edge. The variables of the nearest SPH particle were attributed to each

11 The code, still in development, is not optimized. 


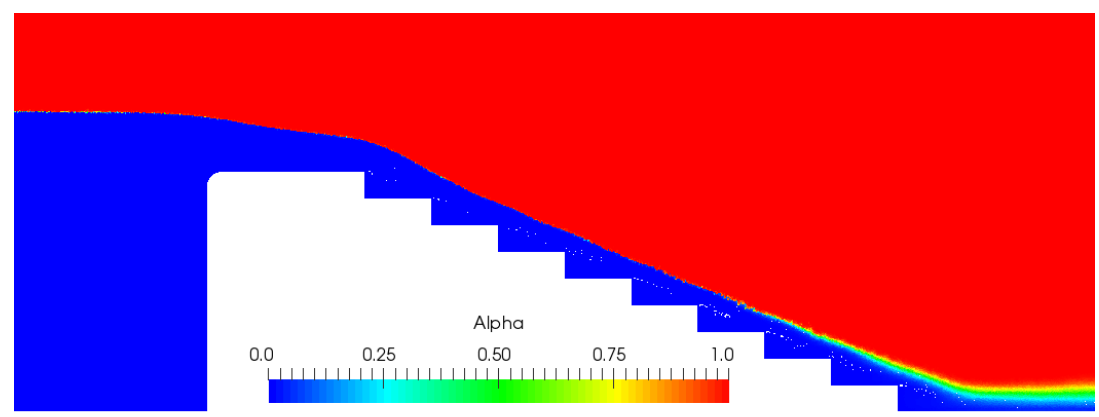

(a) Volume fraction.

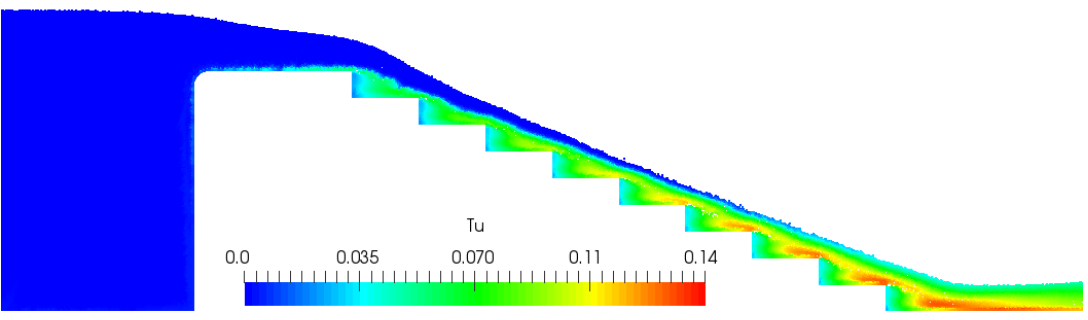

(b) Turbulent intensity (clip for $\alpha<0.9$ ).

Fig. 7: Stepped spillway $-q^{\beta}=0.1819 \mathrm{~m}^{2} / \mathrm{s}$ : air-water mixing at steady state of the skimming regime. The turbulent intensity Tu is computed as $\mathrm{Tu}=\sqrt{2 k / 3} / V_{\max }$ where $V_{\max }$ is the maximum mixture velocity magnitude.

of these points. In order to get smooth profiles for comparison with the timeaveraged experimental results, we proceed to an average of the variables over ten equally-spaced time steps between 8 and $10 \mathrm{~s}$ to get the following results.

For $q^{\beta}=0.1819 \mathrm{~m}^{2} / \mathrm{s}$, Figure 7a displays the volume fraction field over the steps: one can observe that air entrainment occurs just after the sixth step while it has started at this step in the experiments. One can check on Figure $7 \mathrm{~b}$ that it corresponds to the region where the boundary layer is reaching the free surface (for the recall, it is defined here as the isoline $\alpha=0.9$ ), activating the turbulent diffusion term in the relative velocity closure. The pressure field is noisy with pressure waves developing from the step edges and some little voids forming after them in the low pressure region usually prone to cavitation in the absence of air. The background pressure does not appear to be sufficient to prevent the occurrence of those voids. The recirculation velocity field pattern observed in-between the steps is consistent with the experimental observations. The velocity of the flow above the apparent bottom is far larger than the recirculation velocity, so that we focus on the space between the steps in Figure 8. A twice more refined simulation gives us further insights into the entrainment phenomenon as one can identify on 


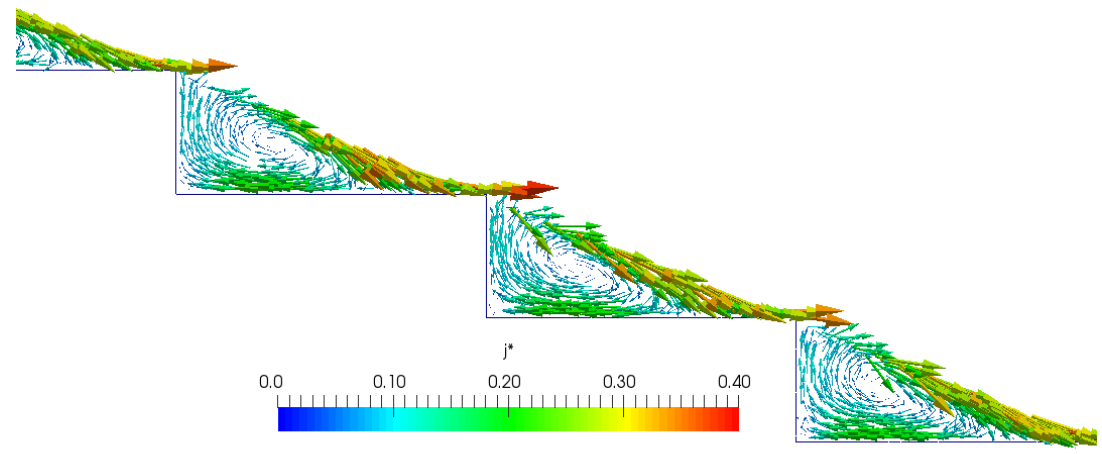

Fig. 8: Stepped spillway: focus on recirculations at the steps 6-8 (nondimensionalization by the maximum velocity).

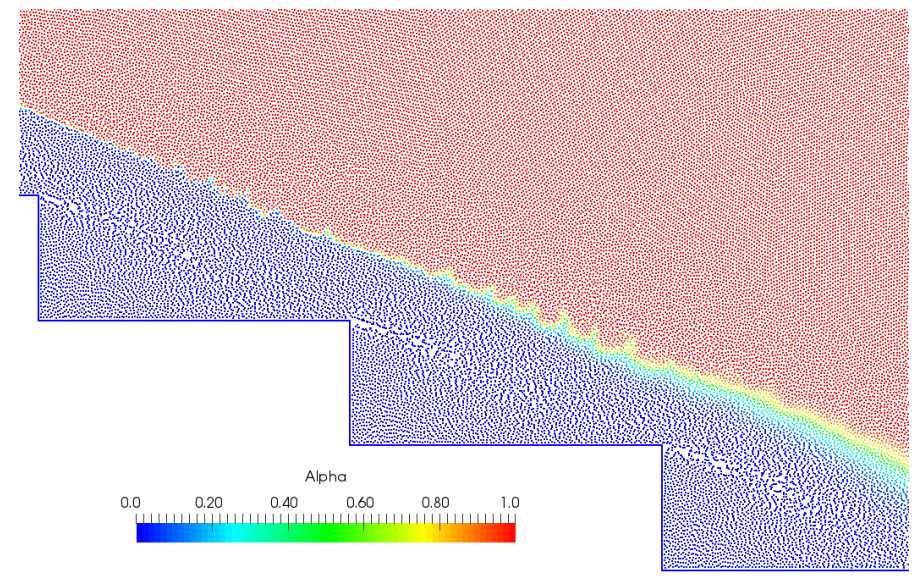

Fig. 9: Stepped spillway $-q^{\beta}=0.1819 \mathrm{~m}^{2} / \mathrm{s}$ : focus on the wavy free surface before entrainment.

Figure 9 small waves developing just before the boundary layer reaching the free surface. A rough assessment of the size of these waves is around $2.5 \mathrm{~cm}$. It is in relative agreement with observations and computations of [60] regarding the Taylor length-scale obtained in an inviscid framework). Those waves do not break as the interface is rapidly blurred by the diffusion of the phases due to the boundary layer reaching the free surface and activating the relative velocity diffusion term. We can relate this waves to the early air entrainment hinted by [60], before the turbulent boundary layer reaching the free surface. Let us have quantitative insights into 


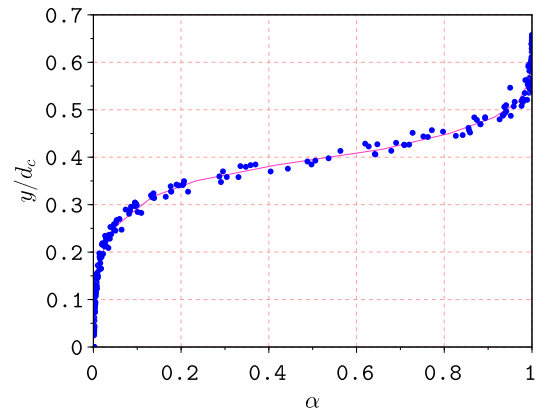

(a) Volume fraction

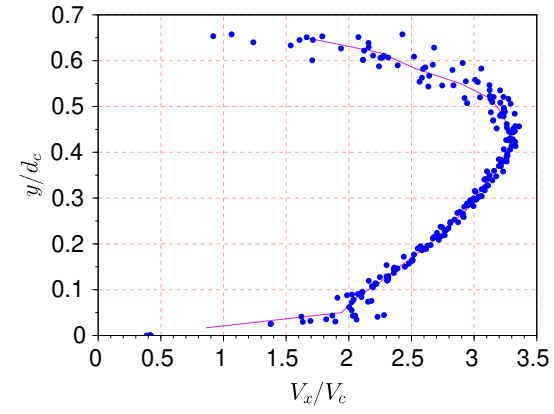

(b) Longitudinal velocity.

Fig. 10: Stepped spillway $-q^{\beta}=0.1819 \mathrm{~m}^{2} / \mathrm{s}$ : variability of the profiles. Symbols: $\mathrm{SPH}$ particles used for the computations. Continuous line: resulting time-averaged value.

the reproduction of the skimming flow. The dispersion of the variables around the averaged values is limited as testified by Figure 10, except near the bottom and in the highly-aerated region. Volume fraction and interfacial air-water velocity profiles at the step edges are displayed on Figures 11a and 11b. In the numerical model, this interfacial velocity is computed as:

$$
V_{x}=\boldsymbol{v}_{i} \cdot \boldsymbol{e}_{x} \quad \text { where } \quad \boldsymbol{v}_{i}=\frac{\boldsymbol{v}^{\alpha}+\boldsymbol{v}^{\beta}}{2}=\boldsymbol{j}+\left(\frac{1}{2}-\alpha\right) \boldsymbol{v}^{r}
$$

The correction linked to the relative velocity proved to be small compared to the mixture velocity.Good shapes are obtained for volume fraction and velocity profiles, even though velocities tend to be over-predicted. The mixing appears to occur later than expected. Profiles are plotted along the direction perpendicular to the slope (coordinate named $y$ ), starting from the edge of each step as indicated on Figure 6 . The uncertainties on the experimental measurements are not detailed for this experimental campaign, but the tools used correspond to the same instruments employed for the measurements of the planar plunging jet detailed in the following section and for which they are detailed [4]:

$$
\left\{\begin{array} { l } 
{ \frac { \Delta \alpha } { \alpha } < \frac { 0 . 0 0 5 } { \alpha } \text { for } \alpha < 0 . 0 5 } \\
{ \frac { \Delta \alpha } { \alpha } < 0 . 0 4 \text { for } 0 . 0 5 < \alpha < 0 . 9 5 } \\
{ \frac { \Delta \alpha } { \alpha } < \frac { 0 . 0 0 2 } { \beta } \text { for } 0 . 9 5 < \alpha }
\end{array} \quad \text { and } \quad \left\{\begin{array}{l}
\frac{\Delta V_{x}}{V_{x}}<0.1 \text { for } 0.01<\alpha<0.05 \\
\frac{\Delta V_{x}}{V_{x}}<0.05 \text { for } 0.05<\alpha<0.95 \\
\frac{\Delta V_{x}}{V_{x}}<0.1 \text { for } 0.95<\alpha<0.99
\end{array}\right.\right.
$$

Let us underline that these uncertainties might be case-dependent. Characteristic air entrainment quantities are displayed on Figure 12. Experimental results were obtained with single (SP) and double tip (DP) probes and one can note some discrepancies between the resulting values, highlighting the dispersion resulting from the unsteadiness and complex topology of the flow. There is a slight underestimation of $Y_{90}$ and $C_{\text {mean }}$ and a slight overestimation of the characteristic velocities. 


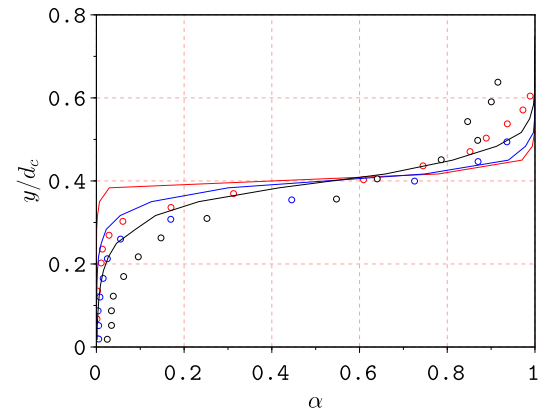

(a) Volume fraction.

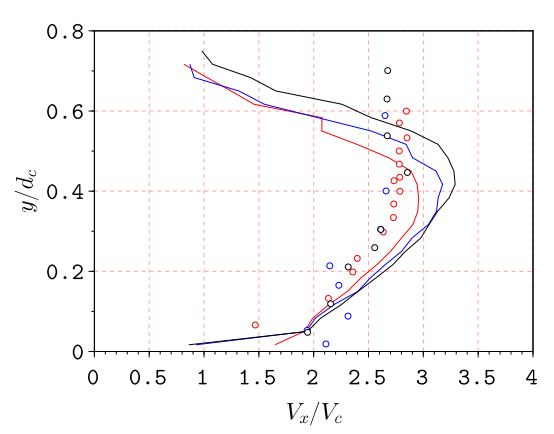

(b) Longitudinal velocity.

Fig. 11: Stepped spillway $-q^{\beta}=0.1819 \mathrm{~m}^{2} / \mathrm{s}$ : vertical distributions above the last three steps (steps 6, 7 and 8 in red, blue and black respectively). Symbols: experimental data by [11]. Continuous lines: present SPH simulation. Dashed lines: analytical solutions based on experimental values detailed in [13].

An interesting point is that it seems to have a one step delay in the profiles and characteristic values, what may point to an inception point appearing too late. Two main actions seem possible to improve the results: modify the size of the dispersed phase or introduce a roughness of the structure. The dispersed phase diameter sensitivity is addressed below. Rough wall laws with different characteristic roughness sizes were tested but did not lead to significant variations of the quantities of interest for physically-based roughness sizes of the order of a few tenths of millimeters. For $q^{\beta}=0.1142 \mathrm{~m}^{2} / \mathrm{s}$, we are in the skimming flow regime. Characteristic air entrainment quantities are displayed on Figure 13. As in the experiment, the inception point is predicted around the fifth step. A reasonable agreement is obtained with the experimental values. The model was also tested for $q^{\beta}=0.058 \mathrm{~m}^{2} / \mathrm{s}$, in the transition flow regime: it is not accurately reproduced due to the strong mixing occurring with the chosen relative velocity closure that assumes that one phase is dispersed. The nappe flow regime, consisting of a succession of falling nappes at each step, is not reproduced for the same reason: we get a continuous mixed flow with intermediate densities that streams over the steps after the first step.

Convergence We performed the simulation $q^{\beta}=0.1819 \mathrm{~m}^{2} / \mathrm{s}$ with three different particle discretizations, by multiplying and dividing by two the reference discretization: no significant variations of the characteristic values were noted as shown on Table 6 .

Sensitivity to the dispersed phase diameter We performed the simulation $q^{\beta}=$ $0.1819 \mathrm{~m}^{2} / \mathrm{s}$ with different sizes of dispersed phase diameters. The influence of the dispersed phase diameter appears to be really limited.

Including the convective transfers The convective transfer term was lately introduced into the set of equations as it triggers some instabilities of the free surface. 


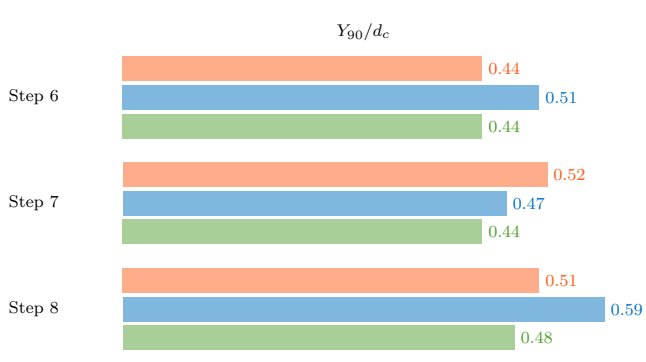

$C_{\text {mean }}$

Step 6
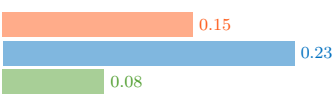

Step 7

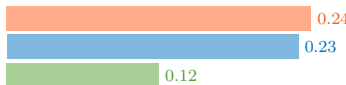

Step 8

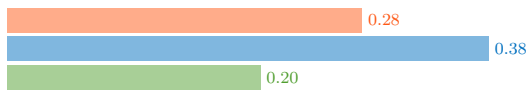

$U_{W} / V_{c}$

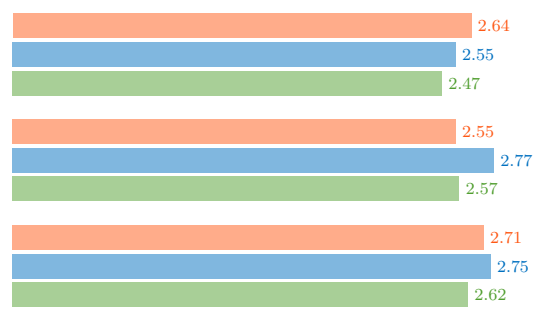

$V_{90} / V_{c}$

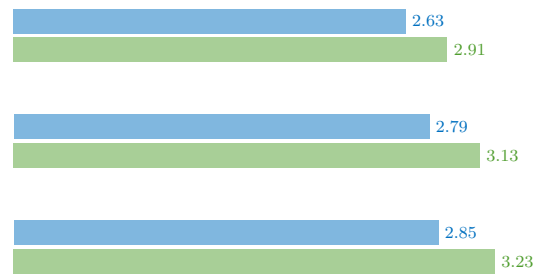

Fig. 12: Stepped Spillway $-q^{\beta}=0.1819 \mathrm{~m}^{2} / \mathrm{s}-$ Characteristic quantities. Present model results in green. Experimental results of [13] with single tip probe in red and double tip probe in blue.

Table 6: Stepped Spillway $-q^{\beta}=0.1819 \mathrm{~m}^{2} / \mathrm{s}-$ Convergence of the characteristic quantities.

\begin{tabular}{|c|c|c|c|c|c|c|c|c|c|c|c|c|}
\hline \multirow{2}{*}{ Step } & \multicolumn{3}{|c|}{$Y_{90} / d_{c}$} & \multicolumn{3}{c|}{$C_{\text {mean }}$} & \multicolumn{3}{c|}{$U_{W} / V_{c}$} & \multicolumn{3}{c|}{$V_{90} / V_{c}$} \\
\cline { 2 - 38 } & $2 \delta r$ & $\delta r$ & $\delta r / 2$ & $2 \delta r$ & $\delta r$ & $\delta r / 2$ & $2 \delta r$ & $\delta r$ & $\delta r / 2$ & $2 \delta r$ & $\delta r$ & $\delta r / 2$ \\
\hline 6 & 0.50 & 0.43 & 0.43 & 0.13 & 0.07 & 0.08 & 2.31 & 2.51 & 2.54 & 2.64 & 2.98 & 2.78 \\
\hline 7 & 0.55 & 0.44 & 0.43 & 0.25 & 0.12 & 0.09 & 2.40 & 2.58 & 2.57 & 2.84 & 3.12 & 2.90 \\
\hline 8 & 0.57 & 0.48 & 0.46 & 0.27 & 0.23 & 0.19 & 2.43 & 2.67 & 2.70 & 3.01 & 3.20 & 3.23 \\
\hline
\end{tabular}

One should take with care the following results: indeed, if they are close to the experimental measurements and correspond to a more rigorous model, one should bear in mind that the agitation partly results from the numerics. In this test case, the flow should be initially perfectly separated, which is not the case because of the slight diffusion of the interface imposed at the initialization and at the open boundaries. For a perfectly separated state, the quantity $\alpha \beta \boldsymbol{v}^{r}$ is null, so that there should not be any contribution of this term in the momentum equation. However, in the simulation, the interface is quickly agitated before the entrainment occurs: this enhances the entrainment phenomenon and triggers also an apparent diffusion for time-averaged value as the separated free surface is moving (this last point can however also happen in the experiments). One can note that the unrest close to the free surface generates higher velocities in this region when the mixture layer is thin.

Pressure gradient in the relative velocity We tested a relative velocity closure without the hydrostatic approximation of the pressure gradient in (23). This is 


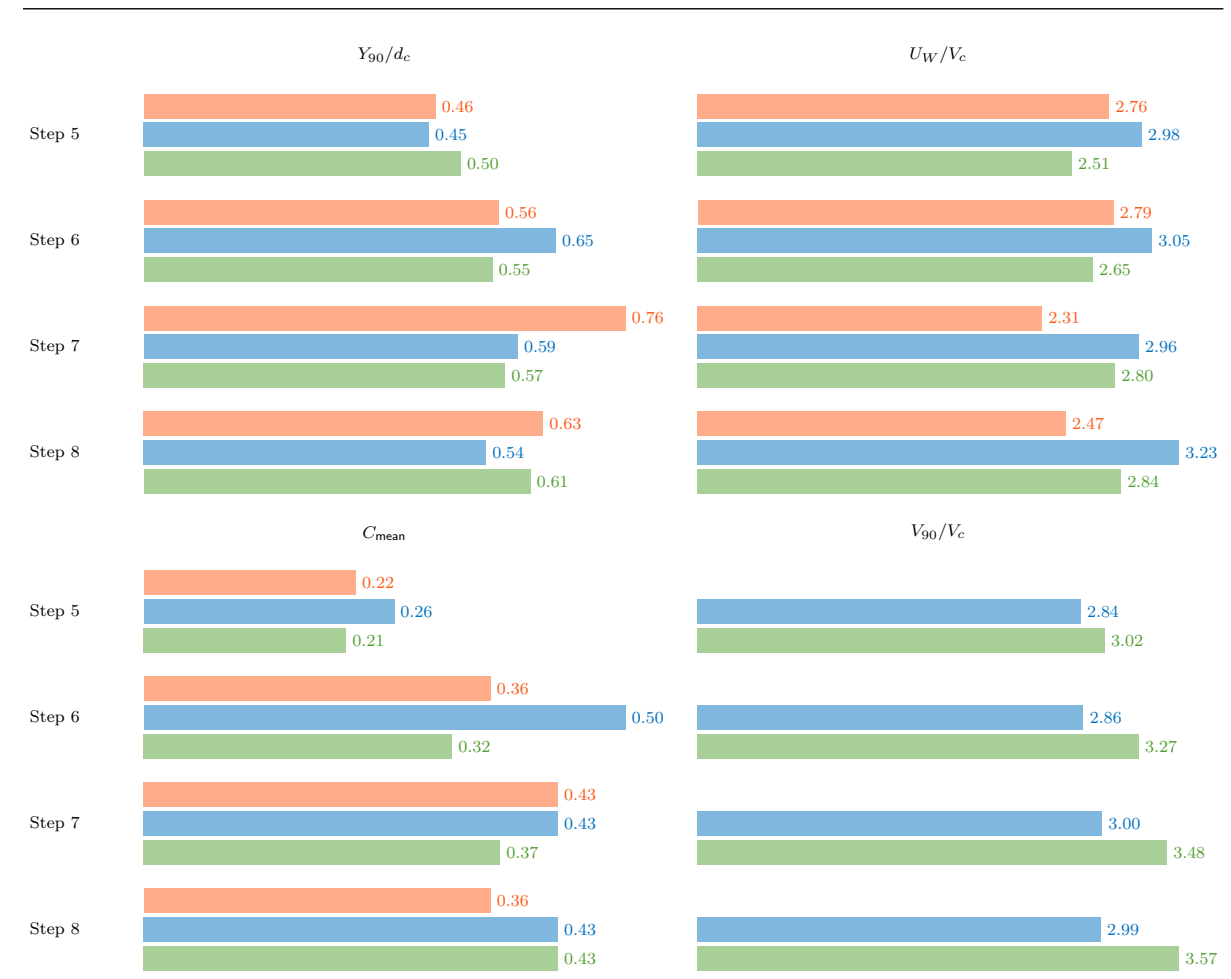

Fig. 13: Stepped Spillway $-q^{\beta}=0.1142 \mathrm{~m}^{2} / \mathrm{s}-$ Characteristic quantities. Present model results in green. Experimental results of [13] with single tip probe in red and double tip probe in blue.

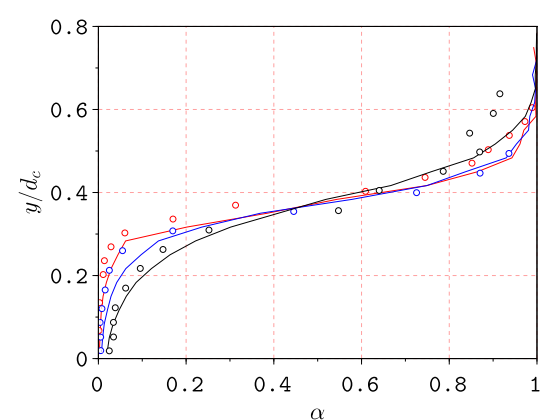

(a) Volume fraction.

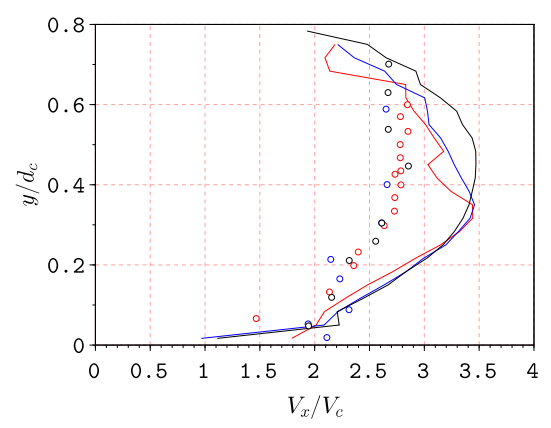

(b) Longitudinal velocity.

Fig. 14: Stepped spillway $-q^{\beta}=0.1819 \mathrm{~m}^{2} / \mathrm{s}$ : including the convective tranfers term, vertical distribution above the last three steps (steps 6,7 and 8 in red, blue and black respectively). Symbols: experimental data by [11]. Continuous lines: present SPH simulation. 
Table 7: Stepped Spillway $-q^{\beta}=0.1819 \mathrm{~m}^{2} / \mathrm{s}-$ Sensitivity to dispersed phase diameter in $\mathrm{mm}$.

\begin{tabular}{|c|c|c|c|c|c|c|c|c|}
\hline & \multicolumn{4}{|c|}{$Y_{90} / d_{c}$} & \multicolumn{4}{|c|}{$C_{\text {mean }}$} \\
\hline Step $d^{\alpha}(\mathrm{mm})$ & 2 & 1 & 0.5 & 0.1 & 2 & 1 & 0.5 & 0.1 \\
\hline 6 & 0.44 & 0.43 & 0.44 & 0.44 & 0.08 & 0.07 & 0.07 & 0.08 \\
\hline 7 & 0.44 & 0.44 & 0.45 & 0.45 & 0.12 & 0.13 & 0.13 & 0.13 \\
\hline \multirow[t]{2}{*}{8} & 0.48 & 0.48 & 0.48 & 0.48 & 0.19 & 0.20 & 0.21 & 0.21 \\
\hline & \multicolumn{4}{|c|}{$U_{W} / V_{c}$} & \multicolumn{4}{|c|}{$V_{90} / V_{c}$} \\
\hline Step $d^{\alpha}(\mathrm{mm})$ & 2 & 1 & 0.5 & 0.1 & 2 & 1 & 0.5 & 0.1 \\
\hline 6 & 2.46 & 2.49 & 2.43 & 2.46 & 2.87 & 2.96 & 2.84 & 2.89 \\
\hline 7 & 2.58 & 2.60 & 2.57 & 2.58 & 3.13 & 3.12 & 3.05 & 3.07 \\
\hline 8 & 2.59 & 2.61 & 2.64 & 2.63 & 3.23 & 3.23 & 3.23 & 3.23 \\
\hline
\end{tabular}

a more-physically based expression that couples completely the system of equations. However, it is complex to handle due to the noisy pressure field encountered. The volume diffusion coefficient was increased from 0.3 to 1 to get a smooth pressure field more adequate for computing its gradient in the relative velocity. The safety factor of the CFL was also divided by two and the maximum relative velocity clipped as pressure waves developed at the first iterations before fading out and led to high values of the relative velocity. The interface is sometimes diffused over a few particles due to some pressure waves propagating along the interface as noted on the previous cases. One can see a very good agreement of the volume fraction profile with experimental values, especially in the dispersed region close to the bottom, highlighting the interest of going towards such physical closure of the relative velocity. No significant gain is obtained for the longitudinal velocity profiles on Figure 15.

\subsection{A local air entrainment test case: the plunging jet}

Plunging jets are rapid flows impinging a slower liquid and belong to the local aeration framework, air being entrained at the intersection between the impinging flow and receiving waters. A review of the literature and basic processes of gas entrainment by plunging jets can be found in $[5,10,32]$. In the following, we consider a planar plunging jet of water entraining air, this case being well-documented in $[9,10,12,7,8,4]$.

Description The geometry is described in Figure 16a with a zoom on the support structure in Figure 16b. One can notice a small angle of the support to the jet chosen to prevent jet detachment. The computational limits drove us to consider the shaded region of Figure 16a. Half of the depth of the domain is modeled and the left-hand side of the domain behind the support is shorter than in the experiment. The flow in the nozzle has not been modeled: a uniform velocity profile is directly imposed on the boundary of the domain to be consistent with the experimental measurements of [4]. The parameters of the model are detailed in Table 8. 


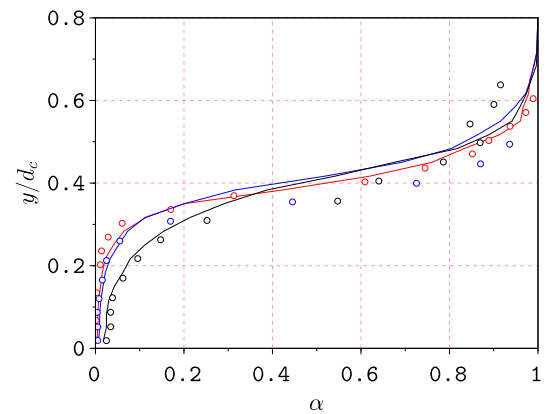

(a) Volume fraction.

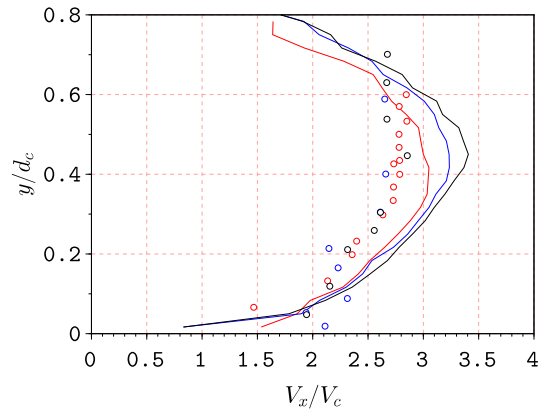

(b) Longitudinal velocity.

Fig. 15: Stepped spillway $-q^{\beta}=0.1819 \mathrm{~m}^{2} / \mathrm{s}$ : with a relative velocity depending on the pressure gradient, vertical distribution above the last three steps (steps 6, 7 and 8 in red, blue and black respectively). Symbols: experimental data by [11]. Continuous lines: present SPH simulation.

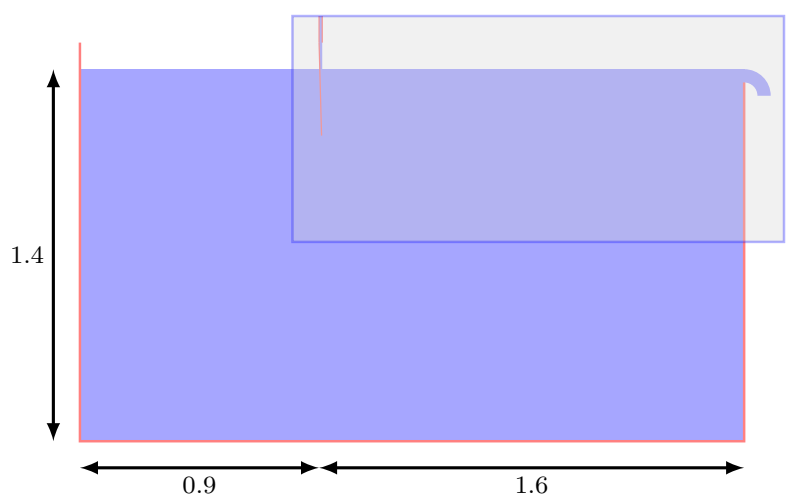

(a) Tank.

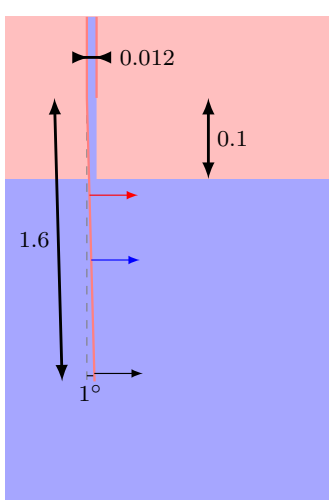

(b) Support structure.

Fig. 16: Planar plunging jet: geometry (distances in $\mathrm{m}$ ). The shaded region corresponds to the numerical domain considered for the computations.

The computations were made without background pressure. The introduction of a background pressure did not prevent jet detachment nor mitigate the appearance of voids within the flow. A volume diffusion coefficient $\Lambda=0.1$ was used. The convective transfer term was not taken into account in the momentum equation. $k-\epsilon$ model was not considered in the following results (the explanation of this choice is detailed in the results part).

Results 1.7 million SPH particles are used. Two weeks of computational time on one graphic card GeForce GTX Titan Black were required for $20 \mathrm{~s}$ of physical time. To compute the profiles along the $y$ direction, a set of points spaced by $\delta r$ were introduced along the horizontal direction starting at the jet support. The variables 
Table 8: Parameters for the planar plunging jet case.

\begin{tabular}{|l|rl|}
\hline$\rho^{\alpha}$ & 1.23 & $\mathrm{~kg} / \mathrm{m}^{3}$ \\
\hline$\nu^{\alpha}$ & $1.5610^{-5}$ & $\mathrm{~m}^{2} / \mathrm{s}$ \\
\hline$c^{\alpha}$ & 45 & $\mathrm{~m} / \mathrm{s}$ \\
\hline$d^{\alpha}$ & 1 & $\mathrm{~mm}$ \\
\hline$\delta r$ & 1 & $\mathrm{~mm}$ \\
\hline
\end{tabular}

\begin{tabular}{|l|rl|}
\hline$\rho^{\beta}$ & 1000 & $\mathrm{~kg} / \mathrm{m}^{3}$ \\
\hline$\nu^{\beta}$ & $10^{-6}$ & $\mathrm{~m}^{2} / \mathrm{s}$ \\
\hline$c^{\beta}$ & 45 & $\mathrm{~m} / \mathrm{s}$ \\
\hline$d^{\beta}$ & 1 & $\mathrm{~mm}$ \\
\hline$p_{B}$ & 0 & $\mathrm{~Pa}$ \\
\hline
\end{tabular}

of the nearest SPH particle were attributed to each of these points. In order to get smooth profiles for comparison with the time-averaged experimental results, we proceed to an average of the variables over equally-spaced time steps between 5 and $11 \mathrm{~s}$ to get the following results (a large period was necessary to decrease the scatter of the resulting averaged profiles). We tested the lowest value of volume flow rate $q^{\beta}=0.0254 \mathrm{~m}^{2} / \mathrm{s}$ (corresponding to the value $Q^{\beta}=q^{\beta} W_{s}=0.00683$ $\mathrm{m}^{3} / \mathrm{s}$ with a width of the jet $\left.W_{s}=0.269 \mathrm{~m}\right)$. We investigate in the following the numerical results and compare them to experimental data of [4].

One can see on Figure 17 the bubble plume with gusts of air packets propagating through vortical structures within the tank. The interface close to the plunging jet is highly agitated. This unrest that certainly affects the air entrainment originates form two sources: the pre-entrainment occurring along the plunging jet that diffuses the interface and increases the region of impact of the jet and the waves propagating in the domain and enhanced by some voids generated in the entrainment region. The two-dimensional framework may prevent some energy dissipation. Such observations were also made by [16] on this test case using a finite volume approach with the NEPTUNE_CFD code. This unrest contributes to significant turbulent kinetic energy production and therefore diffusion of the interface when the $k-\epsilon$ model is used (due to the turbulent diffusion term in the relative velocity diffusion). That is why is was switched off of in the presented results. One can see on Figures $18 \mathrm{a}$ and $18 \mathrm{~b}$, a satisfactory reproduction of the volume fraction profiles and a correct decrease of the longitudinal velocity near the jet support while the decrease in velocity further from the support is not represented correctly by the simulation.

\subsection{A 3D industrial air entrainment case}

We now aim at applying the SPH air-water mixture developed to a three-dimensional industrial test case: a hydraulic discharge-control structure at the La Coche hydraulic power unit as described by [26]. The single-fluid approach proved to fail at reproducing the dynamics of the flow. A two-phase approach appeared to be necessary.

Description As presented on a 2D projection on Figure 19, the structure consists of a drop region where half of the volume flow rate of water falls in the reception tank with vertical plunging jets and the other half flows along the cylinder surrounding this drop region. At the bottom end of this tank, the water exits through a pipe. A critical feature for the design of this structure is the air entrainment and then detrainment within the reception tank to avoid the propagation of air bubbles 


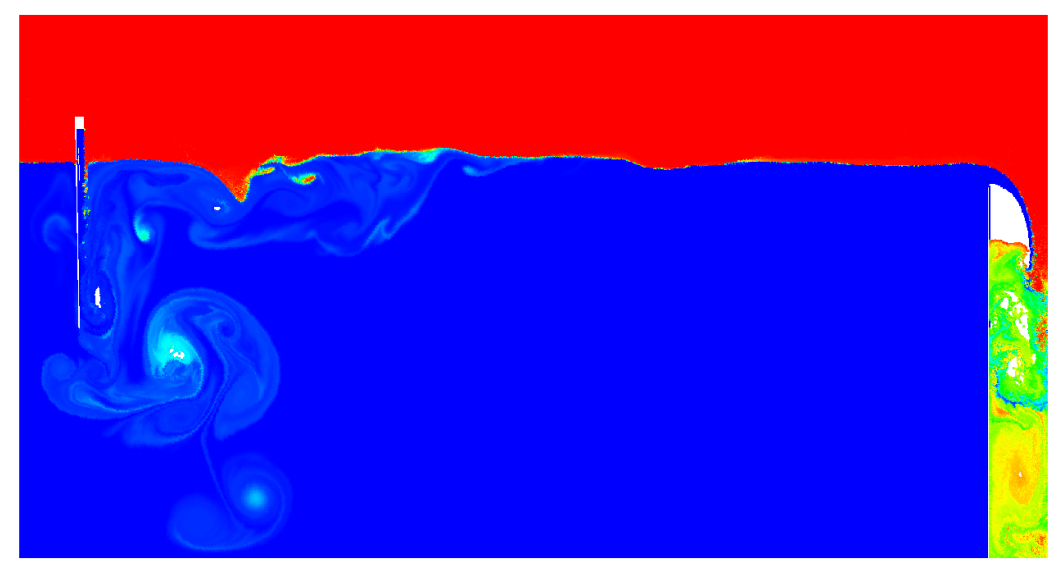

Fig. 17: Planar plunging jet without turbulence model: volume fraction field in larger domain at $t=4 \mathrm{~s}$.

within the pipe. Such structures have already been studied in the literature as done by [34] and illustrate the significant influence that the buoyancy of the entrained air can have on the flow field. The target is two-fold: dissipate a lot of energy thanks to maximized air entrainment in the drop section and remove the maximum of air before entering the pipeline in the detrainment section. Indeed, the formation of air packets within the pipeline could trigger some issues in pumps further down. As shown on Figure 19, depending on the water level in the tank (and therefore the amount of aeration due to the plunging jet), the flow can:

- Go directly to the bottom and reach the pipe without recirculating. A singlephase simulation of this case generates such a flow.

- Rise after the plunging region due to the vertical momentum given by the bubble swarm generated by a strong aeration, and flow along the surface with significant deaeration. Then, getting closer to the pipe outlet, it goes down again, hopefully with little or no aeration. A two-phase approach is required to capture that kind of behavior.

An experimental campaign was carried out on this geometry by the Hydraulic Engineering Center of EDF so that some results are available to compare air concentration and velocity profiles.

The physical and numerical parameters are detailed in Table 9. The simulation counts around 2.76 million particles. Two months of computations were required on one graphic card GeForce GTX Titan Black for $14 s$ of physical time. The $k-\epsilon$ model is used. The convective transfer term was not considered in the momentum equation. The domain is initially at rest, with the tank filled of water at the desired free surface height with an hydrostatic pressure profile. An air layer is put above, with a smooth transition in volume fractions with an hyperbolic tangent function. This air layer does not fill the whole domain due to the construction of 


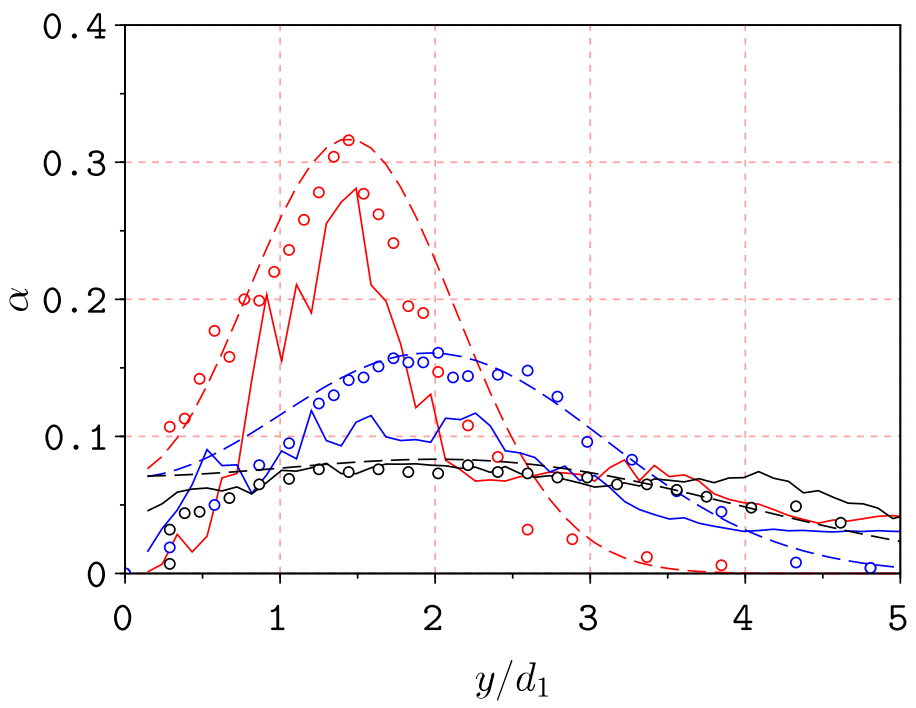

(a) Volume fraction.

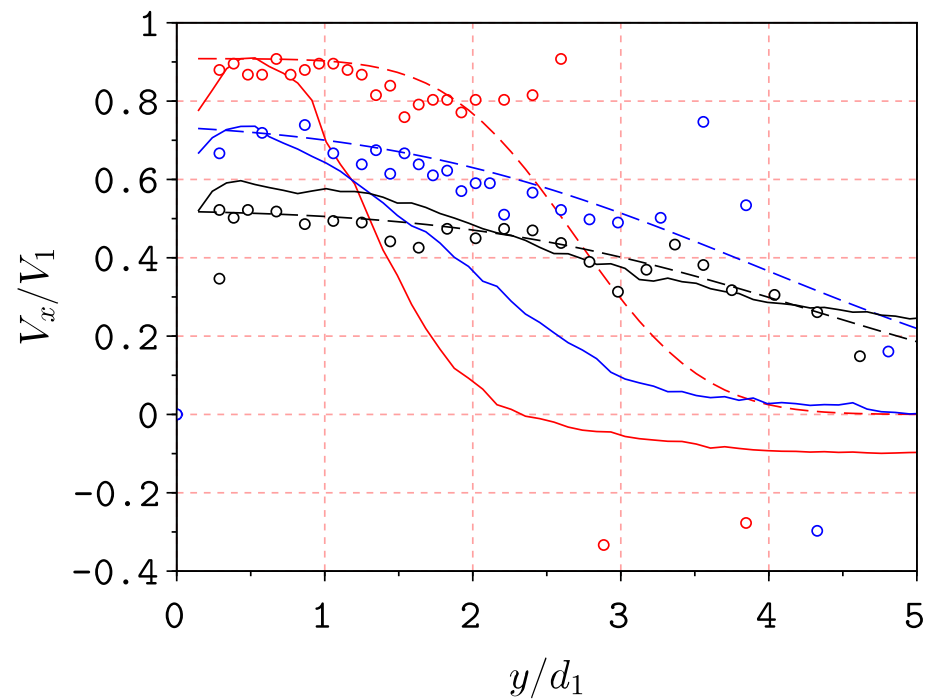

(b) Longitudinal velocity.

Fig. 18: Planar plunging jet - bubble plume: without turbulence model, profiles at distances from the free surface $x-x_{1}=2 \mathrm{~cm}$ in red, $x-x_{1}=10 \mathrm{~cm}$ in blue and $x-x_{1}=24 \mathrm{~cm}$ in black. Symbols: experimental values of [4]. Continuous lines: numerical results. Dashed lines: analytical formulations suggested by [4] based on their measurements. 


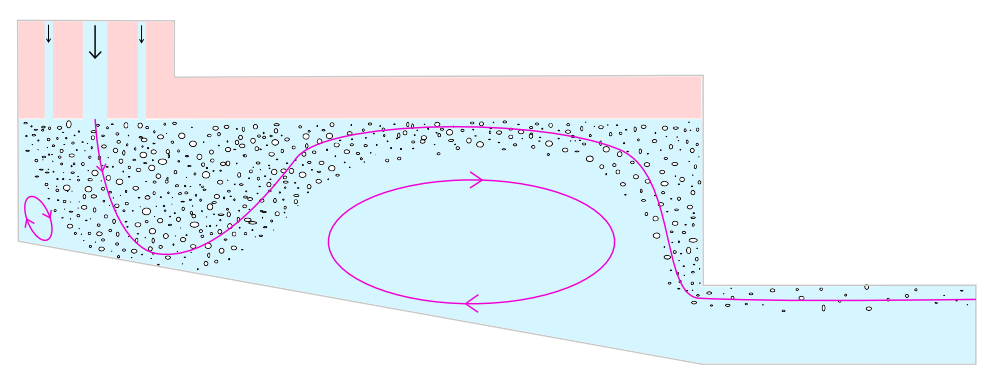

(a) Low tailwater level.

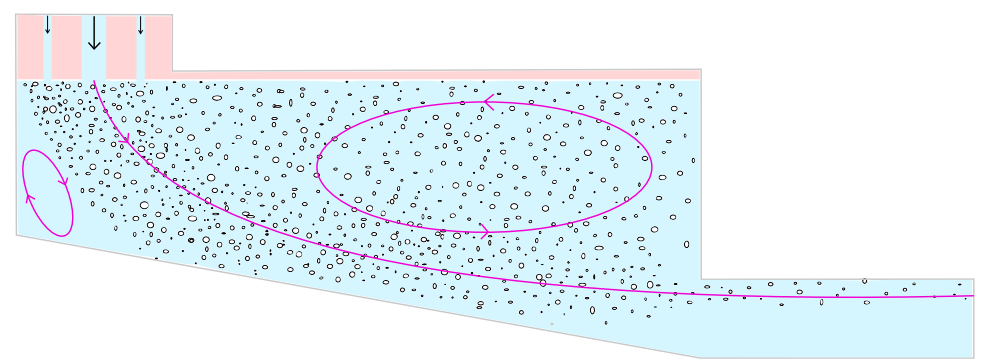

(b) High tailwater level.

Fig. 19: Discharge-control structure: geometry and expected flow patterns.

Table 9: Parameters for the discharge-control structure.

\begin{tabular}{|c|rl|}
\hline$\rho^{\alpha}$ & 1.23 & $\mathrm{~kg} / \mathrm{m}^{3}$ \\
\hline$\nu^{\alpha}$ & $1.5610^{-5}$ & $\mathrm{~m}^{2} / \mathrm{s}$ \\
\hline$c^{\alpha}$ & 40 & $\mathrm{~m} / \mathrm{s}$ \\
\hline$d^{\alpha}$ & 2 & $\mathrm{~mm}$ \\
\hline$g$ & 9.81 & $\mathrm{~m} / \mathrm{s}^{2}$ \\
\hline
\end{tabular}

\begin{tabular}{|l|rl|}
\hline$\rho^{\beta}$ & 1000 & $\mathrm{~kg} / \mathrm{m}^{3}$ \\
\hline$\nu^{\beta}$ & $10^{-6}$ & $\mathrm{~m}^{2} / \mathrm{s}$ \\
\hline$c^{\beta}$ & 40 & $\mathrm{~m} / \mathrm{s}$ \\
\hline$d^{\beta}$ & 2 & $\mathrm{~mm}$ \\
\hline$p_{B}$ & 0 & $\mathrm{~Pa}$ \\
\hline
\end{tabular}

the geometry that did not consider the potential presence of air around the inlet nozzles (absence of separating walls). Only water is used at open boundaries and enters the domain at the inlet at the first iterations. Velocities are imposed with Poiseuille profiles at the inlet pipes. For the turbulent quantities, considering a fully-developed turbulent configuration, the initialization is done with:

$$
k=\frac{3}{2}(U I)^{2} \quad \text { and } \quad \epsilon=C_{\mu}^{\frac{3}{4}} \frac{k^{\frac{3}{2}}}{l}
$$

where $U$ is the average velocity, $I$ the turbulent intensity taken at $10 \%$ and $l$ is the turbulent length scale taken equal to $2 \delta r$. An outlet pipe, further after the exit pipe allows for the flow to exit at the volume flow rate imposed at the inlet through a logarithmic velocity profile.

Results The numerical results are plotted at $\mathrm{t}=14 s$ for half the domain. One can observe the bubble plume on Figure 20. Only small volume fractions reach the 


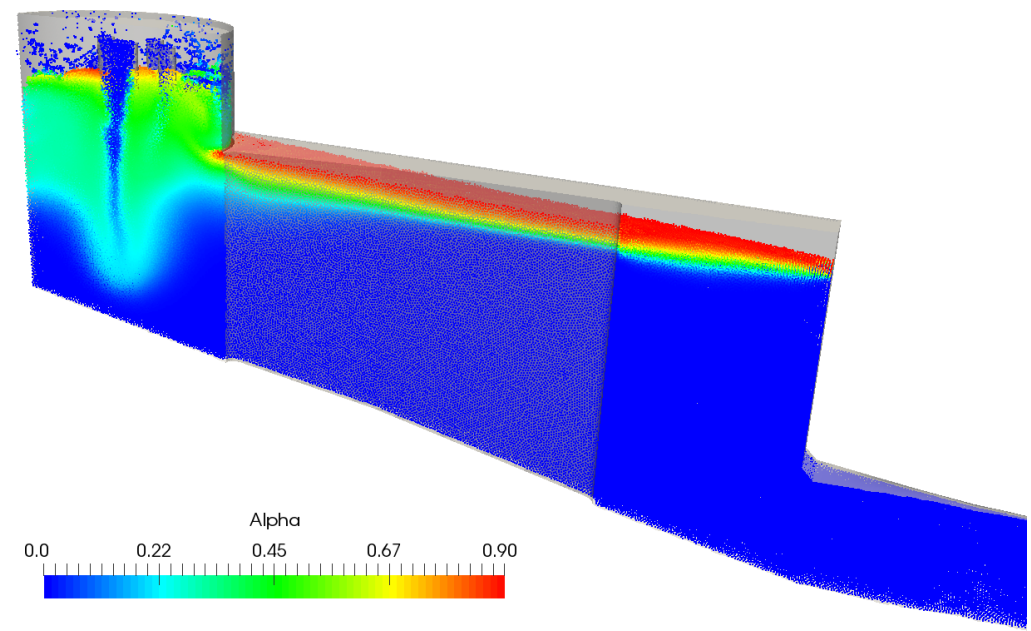

Fig. 20: Discharge-control structure: volume fraction field.

bottom. A region of intense mixing surrounds the inlet jets. Then a bubble swarm propagates with a wavy pattern towards the downstream wall of the tank. No air bubbles were found to be entrained deep enough to reach the outlet pipe at the time considered. Figure 24d highlights the velocity field: one can see that after the initial plunging region, the bubble cloud goes up and entrains water generating a general upward motion of the flow that then goes parallel to the free surface before plunging towards the outlet. A swirling behavior can be noticed in the drop region. A visual comparison of the bubble swarms indicates a satisfactory reproduction of its position and evolution in the basin, with a progressive reduction of its depth towards the downstream wall and some waves propagating along the blurred free surface. Figure 21b displays particles with an air volume fraction smaller than 90\%: above the free surface there is still some mixing due to the relative velocity closure that does not allow a complete separation of phases. We compare in Figure 22 the longitudinal velocity profiles on two different planes detailed in Figure 22a. According to the numerical computations, these velocity profiles are computed in a poorly aerated region. One can see that we significantly underestimate the maximum values. The trend of maximum values at the top of the profiles is reproduced upstream, but the flow appears to plunge earlier towards the exiting pipe in the simulation than in the experiment. We miss the recirculation that is formed before the exit pipe. The significant turbulent kinetic energy in the domain might damp some recirculations due to the increased viscosity. Computations without turbulence model ${ }^{12}$ performed confirmed it: the recirculation going upstream along the bottom of the structure appears, while the flow along the free surface is stronger and plunges more abruptly downwards when reaching the downstream wall. Some very small concentrations of bubbles $(\alpha=0.0001)$ are entrained in the exiting pipe,

12 The planar plunging jet test case suggested that one could get better results for that kind of configuration without the turbulence model considered in this work for local aeration. 


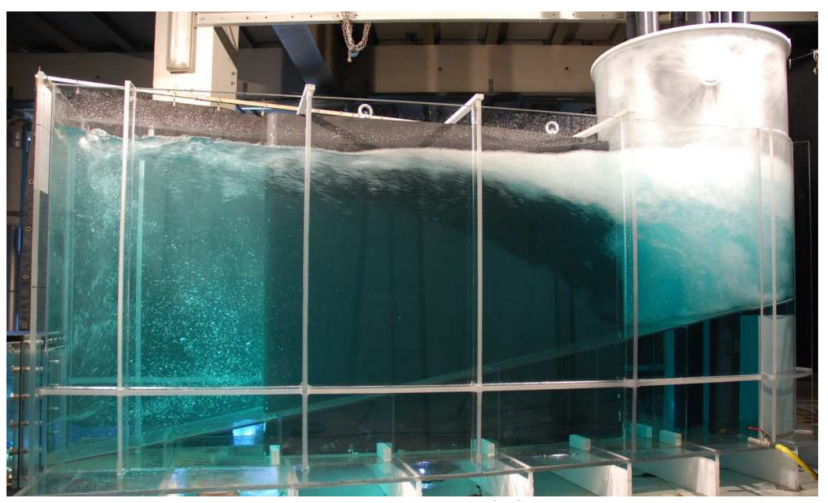

(a) Experiment performed by the Hydraulic Engineering Center (CIH, internal report).

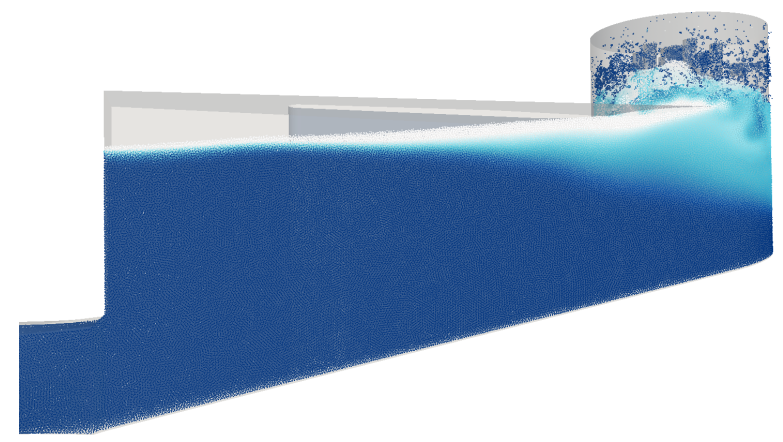

(b) Simulation colored by volume fraction (clip $\alpha<0.9$ ).

Fig. 21: Discharge-control structure: comparison of the bubble swarms.

consistently with some experimental observations on this test case, as shown on Figure $21 \mathrm{~b}$. The velocity profiles at the previously-considered sections averaged on $3 \mathrm{~s}$ are displayed on Figure 23 and highlight a better reproduction of the dynamics of the flow, even though the maximum values are still underestimated.

We compare the velocity magnitude field obtained with our mixture model to the single-fluid formulation on Figures 24a and 24b. With the mixture model, the jet is flapping a little while it falls straight with the single-fluid approach. The rising motion after the jet generated by the bubble swarm that was not caught by the single-fluid formulation is now modeled. One can see that without turbulence model, the dynamics are reproduced better, with the appearance of the recirculations in the reservoir consistently with the experimental observations of Figure 21a. 


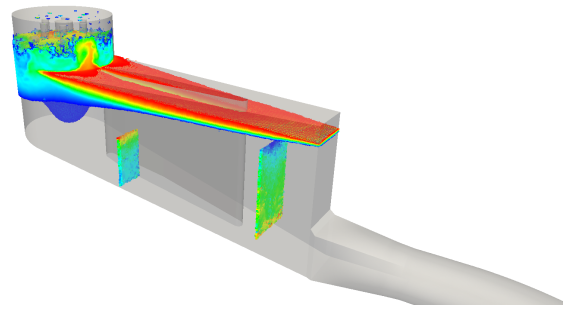

(a) Position of the sections for velocity profiles (the colored volume region corresponds to $0.1<\alpha<0.9)$.
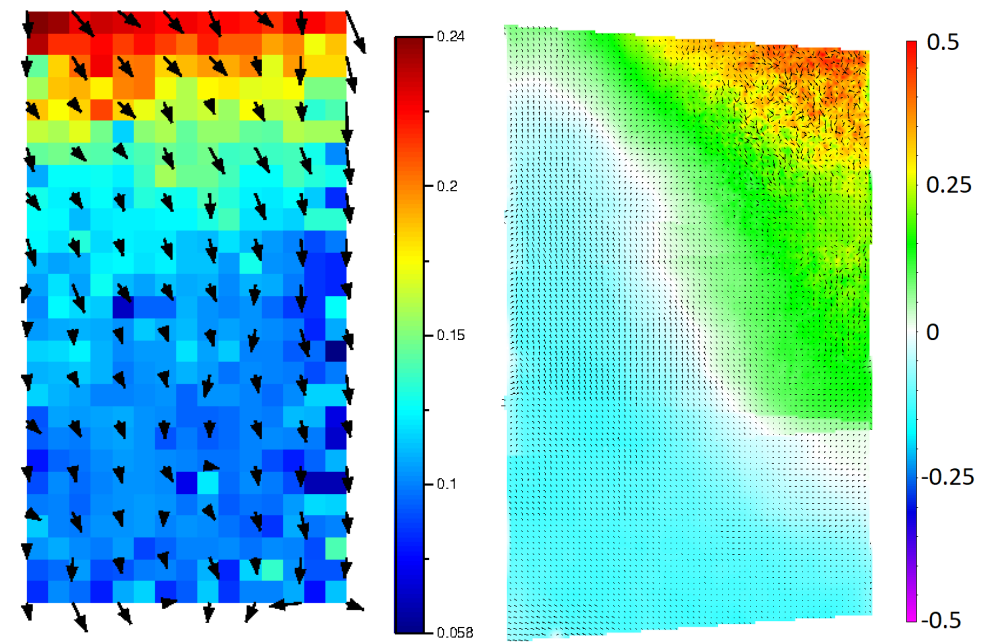

(b) Upstream section: simulation.

(c) Upstream section: experiment.

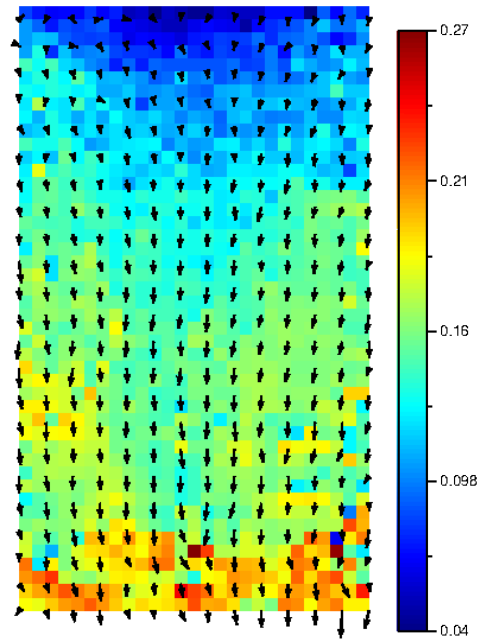

(d) Downstream section: simulation.

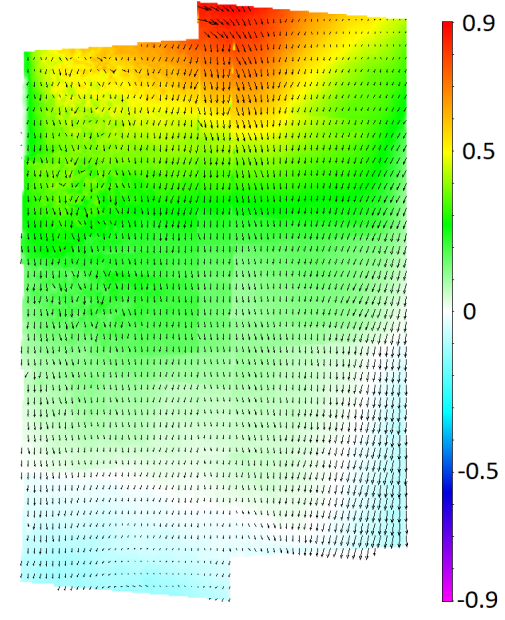

(e) Downstream section: experiment.

Fig. 22: Discharge-control structure: comparison of the longitudinal velocity profiles at two sections. Experiments carried out by the CIH (internal report). 

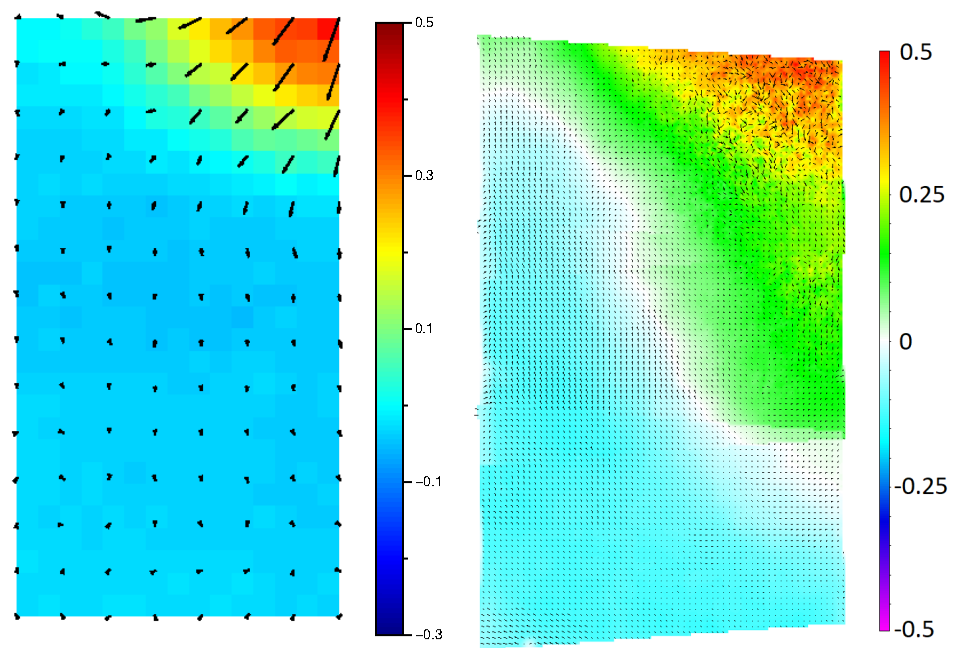

(a) Upstream section: simulation.

(b) Upstream section: experiment.
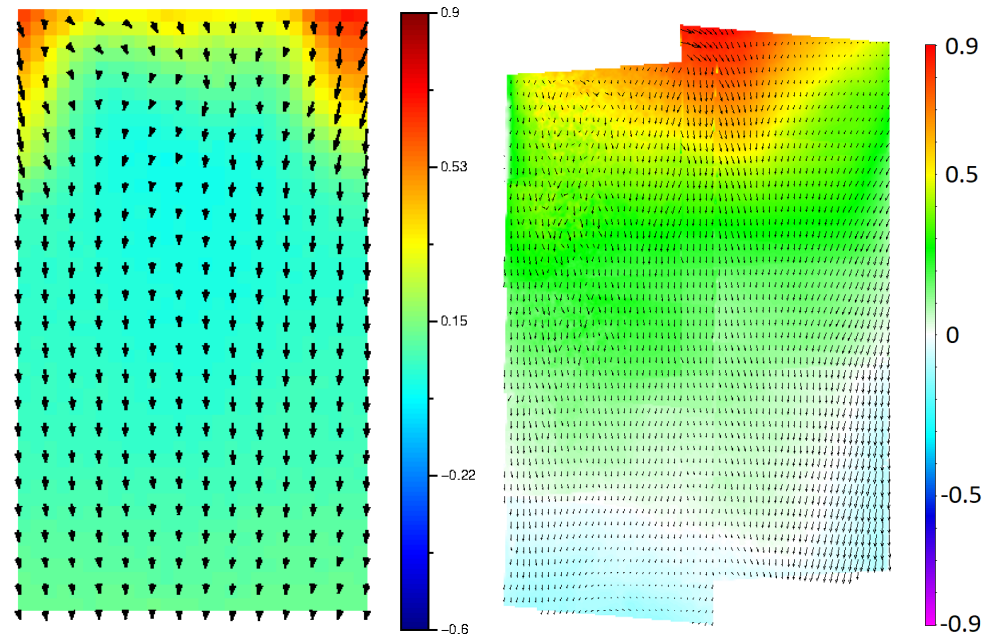

(c) Downstream section: simulation. (d) Downstream section: experiment.

Fig. 23: Discharge-control structure: comparison of the averaged longitudinal velocity profiles at two sections for the computation without turbulence model. Experiments carried out by the CIH (internal report).

\section{Conclusions}

A two-component volume-based SPH mixture model designed to handle high density ratio flows and open boundaries was presented. A relative velocity comprising of a drag and turbulent diffusion parts was introduced. It proves to be a versatile approach, able to deal with different fluids, gases or even sediments [22]. While 


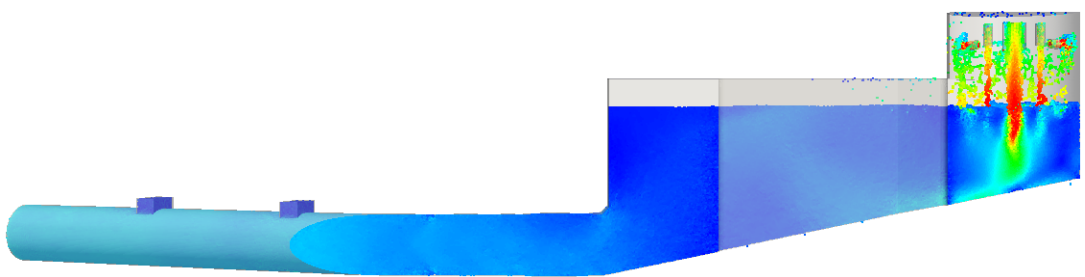

(a) Single-fluid formulation.

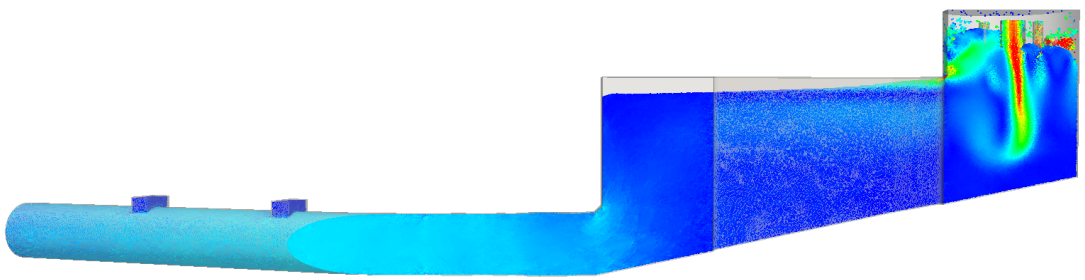

(b) Mixture model formulation (clip $\alpha<0.9)$.

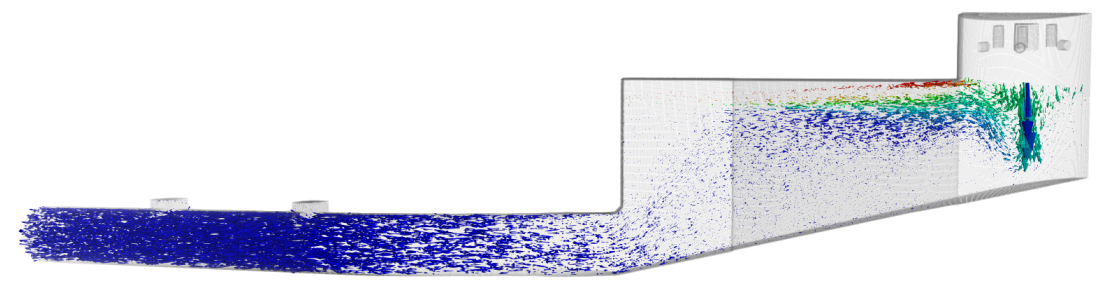

(c) Mixture model formulation with the $k-\epsilon$ turbulence model (clip $\alpha<0.9$ ): velocity vector field colored by $\alpha$.

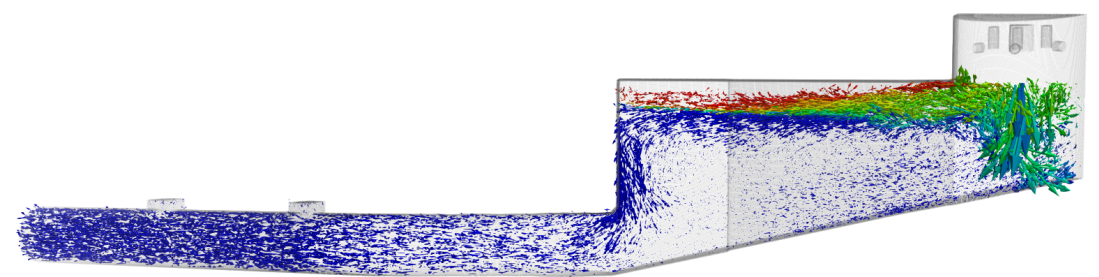

(d) Mixture model formulation without turbulence model (clip $\alpha<0.9$ ): velocity vector field colored by $\alpha$.

Fig. 24: Discharge-control structure: comparison of instantaneous views of the velocity magnitude fields (the absolute velocities might be not comparable).

satisfactory results were obtained for the volume fraction profiles, further work is required to improve the reproduction of two-phase dynamics. In this prospect, investigations shall be carried out on the turbulence modeling, pivotal for those types of applications and the introduction of an SPH formulation ( $\delta-\mathrm{SPH}[27]$, Riemann solver [52]) adapted for high density ratios to improve the robustness of the model and therefore the inclusion of more complex formulations, in particular 
a relative velocity depending on the pressure gradient and the convective transfers in the momentum equation.

\section{Appendices}

\section{A Time integration of the continuity equation}

The following derivation follows faithfully [19], but replacing the density $\rho$ by the inverse of the volume noted $\sigma$, making some adjustments when needed. From the continuity equation (5), one has:

$$
\frac{d \sigma_{a}}{d t}=-\sigma_{a} D_{a}^{\gamma}\{\boldsymbol{j}\}
$$

with:

$$
D_{a}^{\gamma}\{\boldsymbol{j}\}=-\frac{1}{\gamma_{a} \sigma_{a}} \sum_{b \in(\mathcal{F} \cup \mathcal{V})} \theta_{b}\left(\boldsymbol{j}_{a}-\boldsymbol{j}_{b}\right) \cdot \nabla w_{a b}+\frac{1}{\gamma_{a} \sigma_{a}} \sum_{s \in \mathcal{S}} \sigma_{s}\left(\boldsymbol{j}_{a}-\boldsymbol{j}_{s}\right) \cdot \nabla \gamma_{a s}
$$

Let us make the distinction between the Eulerian fluid velocity $\boldsymbol{J}_{b}$ and the Lagrangian particle velocity $\boldsymbol{j}_{b}$. Additional terms then appear in the divergence operator computation and lead to:

$$
\frac{d \sigma_{a}}{d t}=\frac{1}{\gamma_{a}} \sum_{b \in(\mathcal{F} \cup \mathcal{V})} \theta_{b}\left(\boldsymbol{j}_{a}-\boldsymbol{j}_{b}\right) \cdot \nabla w_{a b}-\delta \sigma_{a}^{i / o}-\frac{1}{\gamma_{a}} \sum_{s} \sigma_{s}\left(\boldsymbol{j}_{a}-\boldsymbol{j}_{s}\right) \cdot \boldsymbol{\nabla} \gamma_{a s}+\frac{\sigma_{a}}{\gamma_{a}} \delta \gamma_{a}^{i / o}
$$

With the definitions:

$$
\begin{gathered}
\delta \sigma_{a}^{i / o}=\frac{1}{\gamma_{a}} \sum_{v \in \mathcal{V}^{i / o}} \theta_{v}\left(\boldsymbol{J}_{v}-\boldsymbol{j}_{v}\right) \cdot \nabla w_{a v} \\
\delta \gamma_{a}^{i / o}=\sum_{s \in \mathcal{S}^{i / o}} \frac{\sigma_{s}}{\sigma_{a}}\left(\boldsymbol{J}_{s}-\boldsymbol{j}_{s}\right) \cdot \boldsymbol{\nabla} \gamma_{a s}
\end{gathered}
$$

where $\mathcal{V}^{i / o}$ and $\mathcal{S}^{i / o}$ are respectively the sets of vertex particles and segments belonging to the open boundaries. In a Lagrangian frame, (29) leads to:

$$
\begin{gathered}
\frac{d w_{a b}}{d t}=\left(j_{a}-j_{b}\right) \cdot \nabla w_{a b} \\
\frac{d \gamma_{a}}{d t}=\sum_{s \in \mathcal{S}}\left(j_{a}-j_{s}\right) \cdot \nabla \gamma_{a s}
\end{gathered}
$$

If one makes the approximation:

$$
\frac{d \gamma_{a}}{d t} \approx \sum_{s \in \mathcal{S}} \frac{\sigma_{s}}{\sigma_{a}}\left(\boldsymbol{j}_{a}-\boldsymbol{j}_{s}\right) \cdot \nabla \gamma_{a s}
$$

as volume variations remain limited, (56) now writes:

$$
\frac{d \sigma_{a}}{d t}=\frac{1}{\gamma_{a}} \frac{d}{d t}\left(\sum_{b \in(\mathcal{F} \cup \mathcal{V})} \theta_{b} w_{a b}\right)-\delta \sigma_{a}^{i / o}-\frac{\sigma_{a}}{\gamma_{a}} \frac{d \gamma_{a}}{d t}+\frac{\sigma_{a}}{\gamma_{a}} \delta \gamma_{a}^{i / o}
$$

Hence:

$$
\frac{d}{d t}\left(\gamma_{a} \sigma_{a}\right)=\gamma_{a} \frac{d \sigma_{a}}{d t}+\sigma_{a} \frac{d \gamma_{a}}{d t}=\frac{d}{d t}\left(\sum_{b \in(\mathcal{F} \cup \mathcal{V})} \theta_{b} w_{a b}\right)-\gamma_{a} \delta \sigma_{a}^{i / o}+\sigma_{a} \delta \gamma_{a}^{i / o}
$$


The temporal integration of the continuity equation between $t^{n}$ and $t^{n+1}$ leads to:

$$
\left(\gamma_{a} \sigma_{a}\right)^{n+1}-\left(\gamma_{a} \sigma_{a}\right)^{n}=\sum_{b \in(\mathcal{F} \cup \mathcal{V})}\left(\theta_{b}^{n+1} w_{a b}^{n+1}-\theta_{b}^{n} w_{a b}^{n}\right)-\int_{t^{n}}^{t^{n+1}} \gamma_{a} \delta \sigma_{a}^{i / o}+\int_{t^{n}}^{t^{n+1}} \sigma_{a} \delta \gamma_{a}^{i / o}
$$

With the virtual displacement $\delta r_{a}^{i / o}=\delta t\left(\boldsymbol{J}_{a}^{n}-\boldsymbol{j}_{a}^{n}\right)$, the virtual variations terms write:

$$
\begin{gathered}
\int_{t^{n}}^{t^{n+1}} \gamma_{a} \delta \sigma_{a}^{i / o}=\sum_{v \in \mathcal{V}^{I / O}} \theta_{v}^{n}\left(w\left(\boldsymbol{r}_{a v}^{n}+\delta \boldsymbol{r}_{v}^{i / o}\right)-w\left(\boldsymbol{r}_{a v}^{n}\right)\right) \\
\int_{t^{n}}^{t^{n+1}} \sigma_{a} \delta \gamma_{a}^{i / o}=\frac{1}{2} \sum_{s \in \mathcal{S}^{I / O}} \sigma_{s}^{n}\left(\nabla \gamma_{a s}\left(\boldsymbol{r}_{a s}^{n}+\delta \boldsymbol{r}_{s}^{i / o}\right)+\nabla \gamma_{a s}\left(\boldsymbol{r}_{a s}^{n}\right)\right) \cdot \delta \boldsymbol{r}_{s}^{i / o}
\end{gathered}
$$

where $\delta r_{s}^{i / o}=\delta t\left(\boldsymbol{J}_{s}^{n}-\boldsymbol{j}_{s}^{n}\right)$ and $\delta r_{v}^{i / o}=\delta t\left(\boldsymbol{J}_{v}^{n}-\boldsymbol{j}_{v}^{n}\right)$. In the present work, the factor $\sigma_{s}^{n}$ was approximated by $\sigma_{a}^{n}$, consistently with the approximation (61). For an analytical computation of $\gamma_{a}$ :

$$
\int_{t^{n}}^{t^{n+1}} \sigma_{a} \delta \gamma_{a}^{i / o}=\sum_{s \in \mathcal{S}^{I / O}} \sigma_{s}^{n}\left(\gamma_{a s}\left(\boldsymbol{r}_{a s}^{n}+\delta \boldsymbol{r}_{s}^{i / o}\right)-\gamma_{a s}\left(\boldsymbol{r}_{a s}^{n}\right)\right)
$$

\section{B Riemann problem formulation}

We follow here a similar reasoning to [19]. Neglecting the relative velocity and the viscous efforts at the boundary, the homogeneous (i.e. no relative velocity) mixture model projected along the wall normal ( $n$ index refers to the normal component and $\tau$ index to the tangential component) writes in non-conservative form:

$$
\frac{\partial}{\partial t} \boldsymbol{W}+\boldsymbol{B}(\boldsymbol{W}) \frac{\partial \boldsymbol{W}}{\partial \boldsymbol{n}}=0
$$

where

$$
\boldsymbol{W}=\left(\begin{array}{c}
\sigma \\
\alpha \\
j_{n} \\
j_{\tau}
\end{array}\right) \text { and } \boldsymbol{B}(\boldsymbol{W})=\left(\begin{array}{cccc}
j_{n} & 0 & \sigma & 0 \\
0 & j_{n} & 0 & 0 \\
\frac{c^{2}}{\sigma} & D & j_{n} & 0 \\
0 & 0 & 0 & j_{n}
\end{array}\right)
$$

where $D=\frac{1}{\rho}\left(\rho^{\alpha}\left(c^{\alpha}\right)^{2}-\rho^{\beta}\left(c^{\beta}\right)^{2}\right)\left(\frac{\sigma}{\sigma_{0}}-1\right)$ is deduced from the linearized state law (28). The eigenvalues of $\boldsymbol{B}$ are therefore $j_{n}$ (of multiplicity 2) and $j_{n} \pm c$ where the sound speed writes:

$$
c=\sqrt{\frac{\alpha \rho^{\alpha}\left(c^{\alpha}\right)^{2}+\beta \rho^{\beta}\left(c^{\beta}\right)^{2}}{\alpha \rho^{\alpha}+\beta \rho^{\beta}}}
$$

Following [56], one can compute the associated k-Riemann invariants that are detailed in Table 10 where

$$
\psi(\alpha, \sigma)=c(\alpha) \ln \left(\frac{\sigma}{\sigma_{0}}\right)
$$

As illustrated on Figure 25, depending on the sign of $\lambda_{0}$, the state of the boundary segment is determined by the first or second state. As flows considered are subsonic, $\lambda_{-1}<0$ and $\lambda_{+1}>0 . \lambda_{-1}$ is considered as a ghost wave so that data at exterior state and state 1 are considered to be equal. $\lambda_{0}$ is a contact discontinuity so that the associated $\mathrm{k}$-Riemann invariants hold and simplify in the relations $j_{n, 1}=j_{n, 2}$ and $c\left(\alpha_{1}\right) \ln \left(\frac{\sigma_{1}}{\sigma_{0}}\right)=c\left(\alpha_{2}\right) \ln \left(\frac{\sigma_{2}}{\sigma_{0}}\right)$. To relate the fluid velocity along the normal of the segment and the pressure, one needs to find the relation between state 2 and the interior state (determined through a SPH interpolation with a renormalization by a Shepard filter) across the wave $\lambda_{+1}$. The characteristic wave $\lambda_{+1}$ can correspond to two possible discontinuities: 


$\begin{array}{lccc} & \lambda_{0} & \lambda_{-1} & \lambda_{+1} \\ \alpha & \boldsymbol{X} & \checkmark & \checkmark \\ j_{\tau} & \boldsymbol{X} & \checkmark & \checkmark \\ j_{n}+\psi(\alpha, \sigma) & \checkmark & \checkmark & \boldsymbol{X} \\ j_{n}-\psi(\alpha, \sigma) & \sqrt{ } & \boldsymbol{X} & \sqrt{ }\end{array}$

Table 10: $k$-Riemann invariants for each eigenvalue.

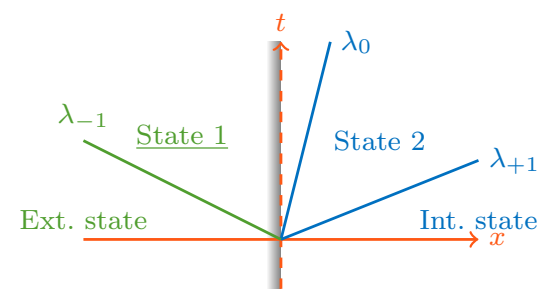

(a) Inlet

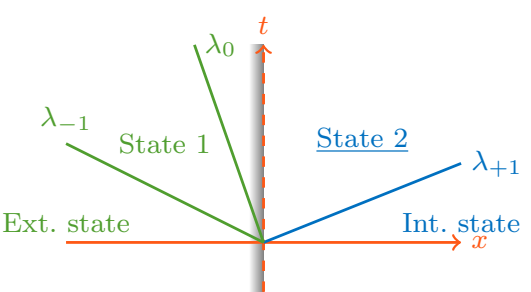

(b) Outlet

Fig. 25: Riemann problems configurations [19]. The state imposed at the boundary is underlined.

- Expansion wave: characteristics are diverging, a smooth transition connects both states and:

$$
\lambda_{+1,2}<\lambda_{+1, \text { int }} \rightarrow \text { Riemann Invariant }
$$

- Shock wave: characteristics are diverging, a smooth transition connects both states and:

$$
\lambda_{+1,2}>\lambda_{+1, \text { int }} \rightarrow \text { Rankine-Hugoniot }
$$

For the shock wave, the Rankine-Hugoniot jump conditions expressed here in terms of conservative variables write:

$$
\begin{gathered}
S\left(\rho_{2}-\rho_{i n t}\right)=\rho_{2} j_{n, 2}-\rho_{i n t} j_{n, i n t} \\
S\left(\rho_{2} \alpha_{2}-\rho_{i n t} \alpha_{i n t}\right)=\rho_{2} \alpha_{2} j_{n, 2}-\rho_{i n t} \alpha_{i n t} j_{n, i n t} \\
S\left(\rho_{2} j_{n, 2}-\rho_{i n t} j_{n, i n t}\right)=p_{2}+\rho_{2} j_{n, 2}^{2}-p_{i n t}-\rho_{i n t} j_{n, i n t}^{2} \\
S\left(\rho_{2} j_{\tau, 2}-\rho_{i n t} j_{\tau, i n t}\right)=\rho_{2} j_{n, 2} j_{\tau, 2}-\rho_{i n t} j_{n, i n t} j_{\tau, i n t}
\end{gathered}
$$

where $S$ is the speed of the shock. Unknown pressure terms are computed thanks to the state equation (28). For the density, we use:

$$
\frac{\rho}{\alpha \rho^{\alpha}+\beta \rho^{\beta}}=\frac{\sigma}{\sigma_{0}}
$$

Combining (73) and (76) with (74), one gets $\alpha_{2}=\alpha_{i n t}$ and $j_{\tau, 2}=j_{\tau, \text { int }}$. Equations (73) and (75) can be combined to determine $\rho_{2}$ and then deduce $u_{n, 2}$ :

$$
\rho_{2} \rho_{i n t}\left(j_{n, 2}-j_{n, i n t}\right)^{2}=\left(\rho_{2}-\rho_{\text {int }}\right)\left(p_{2}-p_{\text {int }}\right)
$$

This relation is explicit if $\rho_{2}$ is known but implicit if $j_{n, 2}$ is known. One should then iterate. Assuming that $\rho$ variations are small, we deduced a first guess from the linearization of the relation that proved to be sufficiently accurate. For the other quantities to compute, at an inlet (i.e. $\lambda_{0}>0$ ), tangential velocity and volume fraction are defined by the user. They are determined from the interior state at an outlet $\left(\right.$ i.e. $\left.\lambda_{0}<0\right)$. In pseudo-code, it leads to the schemes:

- For an imposed velocity: 
if $p_{\text {ext }} \leq p_{\text {int }}$ then

Expansion wave

Riemann invariant $j_{n}+\psi(\alpha, \sigma)$

else

Shock wave

Rankine-Hugoniot relation

end if

States to impose at the interface $i$

if $j_{n, e x t} \geq 0$ then

Inlet: boundary at state 1

$\alpha_{i}=\alpha_{e x t}$

else

Outlet: boundary at state 2

$\alpha_{i}=\alpha_{i n t}$

end if

- For an imposed velocity:

if $j_{n, e x t} \leq j_{n, i n t}$ then

Expansion wave

Riemann invariant $j_{n}+\psi(\alpha, \sigma)$

else

Shock wave

Rankine-Hugoniot relation

end if

States to impose at the interface $i$

if $j_{n, e x t} \geq 0$ then

Inlet: boundary at state 1

$\sigma_{i}$
else

$\sigma_{i}=\sigma_{e x t}$ and $\alpha_{i}=\alpha_{e x t}$

Outlet: boundary at state 2

$\sigma_{i}=\sigma_{2}$ and $\alpha_{i}=\alpha_{\text {int }}$

end if

\section{Analytical solution for the two-phase mixture Poiseuille flow}

At steady state, the volume fraction equation of (6) becomes:

$$
\boldsymbol{\nabla} \cdot\left(\alpha \beta \boldsymbol{v}^{r}\right)=0
$$

Under the longitudinal periodicity condition, it writes:

$$
\frac{d}{d z}\left(\alpha \beta \boldsymbol{v}^{r} \cdot \boldsymbol{e}_{z}\right)=0
$$

The no-flux conditions at the upper and lower walls imply that at steady state the equation (80) becomes after integration:

$$
\boldsymbol{v}^{r} \cdot \boldsymbol{e}_{z}=0
$$

The volume fraction equation will therefore depend on the chosen closure on the relative velocity. Starting with the closure $\boldsymbol{v}^{r}=\boldsymbol{v}_{0}-K \nabla \alpha / \alpha$, the momentum equation of (3) becomes in this framework:

$$
\left\{\begin{array}{l}
\frac{d p}{d z}=-\rho g \\
\frac{d}{d z}\left(\rho \nu \frac{d}{d z} \boldsymbol{j} \cdot \boldsymbol{e}_{x}\right)+\rho \boldsymbol{F} \cdot \boldsymbol{e}_{x}=0
\end{array}\right.
$$

In the simplified momentum equation (82), the dynamic viscosity is variable and depends on the volume fraction solution of equation (81). Let us nondimensionalize the system using $z_{\star}=z / e, j_{\star}=\boldsymbol{j} \cdot \boldsymbol{e}_{x} / U_{m 0}$ with $U_{m 0}=\rho^{\beta} F e^{2} /\left(3 \mu^{\beta}\right)$ (discharge for the usual single-fluid Poiseuille flow $), p_{\star}=p /\left(\rho^{\beta}\right.$ ge $)$ and introduce the Péclet number $\mathrm{Pe}=e\left|\boldsymbol{v}_{0} \cdot \boldsymbol{e}_{z}\right| / K$ as the 
ratio of convective and diffusive transports. Noting the density ratio $R_{\rho}=\left(\rho^{\alpha}-\rho^{\beta}\right) / \rho^{\beta}$ and viscosity ratio $R_{\mu}=\left(\mu^{\alpha}-\mu^{\beta}\right) / \mu^{\beta}$, the system becomes:

$$
\left\{\begin{array}{l}
\frac{d \alpha}{d z_{\star}}=\operatorname{Pe} \alpha \\
\frac{d p_{\star}}{d z_{\star}}=-\left(1+R_{\rho} \alpha\right) \\
\frac{d}{d z_{\star}}\left[\left(1+R_{\mu} \alpha\right) \frac{d j_{\star}}{d z_{\star}}\right]=-3\left(1+R_{\rho} \alpha\right)
\end{array}\right.
$$

Constant dynamic viscosity The nondimensionalized solution for a constant dynamic viscosity $\mu=\mu^{\alpha}=\mu^{\beta}$ writes:

- Volume fraction

- Pressure profile

$$
\alpha\left(z_{\star}\right)=\alpha_{1} \exp \left(\operatorname{Pe} z_{\star}\right)
$$

$$
p_{\star}\left(z_{\star}\right)=p_{B \star}+1-z_{\star}+\alpha_{1} R_{\rho} \frac{\exp (\mathrm{Pe})}{\mathrm{Pe}}\left[1-\exp \left(-\mathrm{Pe}\left(1-z_{\star}\right)\right)\right]
$$

where $p_{B \star}$ is the background pressure nondimensionalized as the total pressure.

- Longitudinal velocity

$$
\begin{aligned}
j_{\star}\left(z_{\star}\right)= & \frac{3}{2}\left(1-z_{\star}^{2}\right)+\frac{3}{\mathrm{Pe}^{2}}\left[\operatorname{Li}_{2, R}\left(z_{\star}\right)+\mathrm{Pe} z_{\star} \ln _{R}\left(z_{\star}\right)\right. \\
& \left.-\frac{r}{R} \ln _{R}\left(z_{\star}\right)+C_{1}\left(\ln _{R}\left(z_{\star}\right)-\operatorname{Pe} z_{\star}\right)-C_{2}\right]
\end{aligned}
$$

where $\mathrm{Li}_{2}$ is the dilogarithm function that writes $\operatorname{Li}_{2}(x)=-\int_{0}^{x} \ln (1-t) / t d t[1]$. If $|x| \leq$ 1 , one can write a series expression $\mathrm{Li}_{2}(x)=\sum_{n} x^{n} / n^{2}$. We introduced the notations $\ln _{R}\left(z_{\star}\right)=\ln \left(1+\alpha_{1} R_{\mu} \exp \left(\operatorname{Pe} z_{\star}\right)\right)$ and $\operatorname{Li}_{2, R}\left(z_{\star}\right)=\mathrm{Li}_{2}\left(-\alpha_{1} R_{\mu} \exp \left(\mathrm{Pe} z_{\star}\right)\right) . C_{1}$ and $C_{2}$ are deduced from the no-slip condition at walls:

$$
\begin{gathered}
C_{1}=\frac{\operatorname{Li}_{2, R}(1)-\mathrm{Li}_{2, R}(-1)+\mathrm{Pe}\left[\ln _{R}(1)+\ln _{R}(-1)\right]-R_{\rho} / R_{\mu}\left[\ln _{R}(1)-\ln _{R}(-1)\right]}{2 \mathrm{Pe}-\ln _{R}(1)+\ln _{R}(-1)} \\
C_{2}=\mathrm{Li}_{2, R}(1)+\mathrm{Pe} \ln _{R}(1)-\frac{R_{\rho}}{R_{\mu}} \ln _{R}(1)+C_{1}\left(\ln _{R}(1)-\mathrm{Pe}\right)
\end{gathered}
$$

$\alpha_{1}$ is computed thanks to the conservation of volume (integrating over the height of the channel) for a given initial uniform profile of $\alpha(z)=\alpha_{0}$ :

$$
\frac{\alpha_{1}}{\alpha_{0}}=\frac{\mathrm{Pe}}{\sinh (\mathrm{Pe})}
$$

To avoid complete separation of phases, (84) gives a condition on $\alpha_{1}$ :

$$
0 \leq \alpha_{1} \leq \exp (-\mathrm{Pe})
$$

And therefore a condition on the initial uniform volume fraction $\alpha_{0}$ using (89):

$$
0 \leq \alpha_{0} \leq \frac{1-\exp (-2 \mathrm{Pe})}{2 \mathrm{Pe}}
$$

\section{Compliance with Ethical Standards}

This study was funded by EDF R\&D and the French Research Agency (CIFRE grant number \#2016-0362). 


\section{Conflict of interest}

The authors declare that they have no conflict of interest.

\section{References}

1. Abramowitz, M., Stegun, I.: Handbook of Mathematical Functions with Formulas, Graphs, and Mathematical Tables. U.S. Department of Commerce (1964)

2. Alvarado-Rodríguez, C.E., Klapp, J., Sigalotti, L.D.G., Domínguez, J.M., de la Cruz Sánchez, E.: Nonreflecting outlet boundary conditions for incompressible flows using SPH. Computers \& Fluids 159, 177-188 (2017). DOI 10.1016/j.compfluid.2017.09.020

3. Bertevas, E., Tran-Duc, T., Khoo, B.C., Phan-Thien, N.: Smoothed Particle Hydrodynamics (SPH) Applications in Some Sediment Dispersion Problems. In: Proceedings of the 7th International Conference on Computational Methods (ICCM2016). Berkeley, California, United States (2016)

4. Bertola, N.J., Wang, H., Chanson, H.: Air bubble entrainment at vertical plunging jets: a large-scale experimental study. Tech. rep., School of Civil Engineering, The University of Queensland (2017). Hydraulic Model Report CH, 104/17

5. Biń, A.: Gas entrainment by plunging liquid jets. Chemical Engineering Science 48(21), 3585-3630 (1993). DOI 10.1016/0009-2509(93)81019-R

6. Blondel, F., Audebert, B., Pasutto, T., Stanciu, M.: Condensation models and boundary conditions for non-equilibrium wet steam flows. International Journal on Finite Volumes $10(2013)$

7. Brattberg, T., Chanson, H.: Air entrapment and air bubble dispersion at two-dimensional plunging water jets. Chemical Engineering Science 53(24), 4113-4127 (1998). DOI 10.1016/S0009-2509(98)80004-3

8. Brattberg, T., Chanson, H., Toombes, L.: Experimental Investigations of Free-Surface Aeration in the Developing Flow of Two-Dimensional Water Jets. Journal of Fluids Engineering 120(4), 738-744 (1998). DOI 10.1115/1.2820731

9. Chanson, H.: Air Bubble Diffusion in Supercritical Open Channel Flows. In: Proceedings of the 12th Australasian Fluid Mechanics Conference. Sydney, Australia (1995)

10. Chanson, H.: Air Bubble Entrainment in Free-Surface Turbulent Shear Flows. Academic Press (1996). DOI 10.1016/B978-0-12-168110-4.X5000-0

11. Chanson, H.: Caractéristiques diphasiques des écoulements sur les coursiers en marches d'escalier. La Houille Blanche 8, 16-28 (2001). DOI 10.1051/lhb/2001084

12. Chanson, H., Brattberg, T.: Air entrainment by two-dimensional plunging jets: the impingement region and the very-near flow field. In: Proceedings of the 1998 ASME Fluids Engineering Division Summer Meeting. Washington DC, United States (1998)

13. Chanson, H., Toombes, L.: Experimental Investigations of Air Entrainment in Transition and Skimming Flows down a Stepped Chute. Application to Embankment Overflow Stepped Spillways. Tech. Rep. CE158, Department of Civil Engineering, The University of Queensland, Brisbane, Australia (2001)

14. Clift, R., Grace, J.R., Weber, M.E.: Bubbles, Drops and Particles. Dover (1978)

15. Cueille, P.V.: Modélisation par Smoothed Particle Hydrodynamic des phénomènes de diffusion présents dans un écoulement. Ph.D. thesis, INSA de Toulouse (2005). Available at core.ac.uk/download/pdf/35285308.pdf

16. Denèfle, R.: Modélisation locale diphasique eau-vapeur des écoulements dans les générateurs de vapeur. Ph.D. thesis, Université Bordeaux 1 (2013). Available at orioai.u-bordeaux1.fr/pdf/2013/DENEFLE_ROMAIN_2013.pdf

17. Douillet-Grellier, T., Vuyst, F.D., Calandra, H., Ricoux, P.: Simulations of intermittent two-phase flows in pipes using smoothed particle hydrodynamics. Computers \& Fluids 177, 101-122 (2018). DOI 10.1016/j.compfluid.2018.10.004

18. Español, P., Revenga, M.: Smoothed dissipative particle dynamics. Physical Review E 67 (2003). DOI 10.1103/PhysRevE.67.026705

19. Ferrand, M., Joly, A., Kassiotis, C., Violeau, D., Leroy, A., Morel, F.X., Rogers, B.D.: Unsteady open boundaries for SPH using semi-analytical conditions and Riemann solver in 2D. Computer Physics Communications 210, 29-44 (2017). DOI 10.1016/j.cpc.2016.09.009 
20. Ferrand, M., Laurence, D.R., Rogers, B.D., Violeau, D., Kassiotis, C.: Unified semianalytical wall boundary conditions for inviscid, laminar or turbulent flows in the meshless SPH method. International Journal for Numerical Methods in Fluids 71, 446-472 (2013). DOI 10.1002/fld.3666

21. Fonty, T., Ferrand, M., Leroy, A., Joly, A., Violeau, D.: An upwind scheme for conservative, realizable two-phase mixture SPH model with high density ratios. In: Proceedings of 13 th international SPHERIC workshop. Galway, Ireland (2018)

22. Fonty, T., Ferrand, M., Leroy, A., Joly, A., Violeau, D.: Mixture model for twophase flows with high density ratios: a conservative and realizable SPH formulation. International Journal of Multiphase Flow 111, 158-174 (2019). DOI 10.1016/j.ijmultiphaseflow.2018.11.007

23. Ghaïtanellis, A., Violeau, D., Ferrand, M., Abderrezzak, K.E.K., Leroy, A., Joly, A.: A SPH elastic-viscoplastic model for granular flows and bed-load transport. Advances in Water Resources 111, 156-173 (2018). DOI 10.1016/j.advwatres.2017.11.007

24. Grenier, N., Antuono, M., Colagrossi, A., Touzé, D.L., Alessandrini, B.: An Hamiltonian interface SPH formulation for multi-fluid and free surface flows. Journal of Computational Physics 228(22), 8380-8393 (2009). DOI 10.1016/j.jcp.2009.08.009

25. Grenier, N., Touzé, D.L., Colagrossi, A., Colicchio, G., Antuono, M.: SPH multiphase simulation of bubbly flows. Towards oil and water separation. In: Proceedings of the ASME 2013 32nd International Conference on Ocean, Offshore and Arctic Engineering OMAE 2013, vol. 7. Nantes, France (June 9-14, 2013). DOI 10.1115/OMAE2013-11610

26. Guyot, G., Rodriguez, M.: La Coche Pelton enhancement project scale model. In: Proceedings of the 37th IAHR World Congress. Kuala Lumpur, Malaysia (2017)

27. Hammani, I., Oger, G., Touzé, D.L., Colagrossi, A., Marrone, S.: How to derive the multifluid delta-SPH model. In: Proceedings of the 13th International SPHERIC Workshop. Galway, Ireland (2018)

28. Hirt, C.W.: Modeling turbulent air entrainment of air at a free surface. Tech. rep., Flow Science, Inc (2003)

29. Hu, X.Y., Adams, N.A.: A multi-phase SPH method for macroscopic and mesoscopic flows. Journal of Computational Physics 213, 844-861 (2006). DOI 10.1016/j.jcp.2005.09.001

30. Hu, X.Y., Adams, N.A.: An incompressible multi-phase SPH method. Journal of Computational Physics 227, 264-278 (2007). DOI 10.1016/j.jcp.2007.07.013

31. Ishii, M., Hibiki, T.: Thermo-fluid dynamics of two-phase flow - Second Edition. Springer (2011)

32. Kiger, K., Duncan, J.H.: Air-Entrainment Mechanisms in Plunging Jets and Breaking Waves. Annual Review of Fluid Mechanics 44(06), 563-596 (2012). DOI 10.1146/annurevfluid-122109-160724

33. Kobus, H.: Local air entrainment and detrainment. In: Symposium on Scale Effects in Modelling Hydraulic Structures. Stuttgart, Germany (1984). DOI 10.18419/opus-559

34. Kobus, H., Westrich, B.: An example of a combined disharge-control and aeration structure. In: Proceedings of the 20th Congress of the IAHR. Moscow, USSR (1983)

35. Kruisbrink, A.C.H., Pearce, F.R., Yue, T., Morvan, H.P.: An SPH multi-fluid model based on quasi buoyancy for interface stabilization up to high density ratios and realistic wave speed ratios. International Journal for Numerical Methods in Fluids 87(10), 487-507 (2018). DOI 10.1002/fld.4498

36. Kunz, P., Hirschler, M., Huber, M., Nieken, U.: Inflow/outflow with Dirichlet boundary conditions for pressure in ISPH. Journal of Computational Physics 326, 171-187 (2016). DOI 10.1016/j.jcp.2016.08.046

37. Labois, M.: Modélisation des déséquilibres mécaniques dans les écoulements diphasiques : approches par relaxation et par modèle réduit. Ph.D. thesis, Université de Provence, Marseille (2008). Pp. 24-27. Available at tel.archives-ouvertes.fr/tel-00338818

38. Lastiwka, M., Basa, M., Quinlan, N.J.: Permeable and non-reflecting boundary conditions in SPH. International Journal for Numerical Methods in Fluids 61(7), 709-724 (2009). DOI 10.1002/fld.1971

39. Launder, B., Spalding, D.B.: The Numerical Computation of Turbulent Flow. Computer Methods in Applied Mechanics and Engineering 3(2), 269-289 (1974). DOI 10.1016/00457825(74)90029-2

40. Leroy, A.: Un nouveau modèle SPH incompressible : vers l'application à des cas industriels. Ph.D. thesis, Université Paris Est (2014). Available at pastel.archives-ouvertes.fr/tel01126905 
41. Leroy, A., Violeau, D., Ferrand, M., Kassiotis, C.: Unified semi-analytical wall boundary conditions applied to 2-d incompressible SPH. Journal of Computational Physics 261, 106-129 (2014). DOI 10.1016/j.jcp.2013.12.035

42. Li, S., Zhang, J., Xu, W.: Numerical investigation of air-water flow properties over steep float and pooled stepped spillways. Journal of Hydraulic Research 56(1), 1-14 (2018). DOI 10.1080/00221686.2017.1286393

43. Manninen, M., Taivassalo, V.: On the mixture model for multiphase flow, vol. 288. Technical Research Center of Finland (1996)

44. Morris, J.P., Fow, P.J., Zhu, Y.: Modeling low Reynolds number incompressible flows using SPH. Journal of Computational Physics 136(1), 214-226 (1997). DOI 10.1006/jcph.1997.5776

45. Nikseresht, A.H., Talebbeydokhti, N., Rezaei, M.J.: Numerical simulation of twophase flow on step-pool spillways. Scientia Iranica 20(2), 222-230 (2013). DOI 10.1016/j.scient.2012.11.013

46. Oger, G.: Aspects théoriques de la méthode SPH et applications à l'hydrodynamique à surface libre. Ph.D. thesis, École Centrale de Nantes (2006). Available at docplayer.fr/54160459-Aspects-theoriques-de-la-methode-sph-et-applications-a-lhydrodynamique-a-surface-libre-guillaume-oger.html

47. Pope, S.B.: Turbulent flows. Cambridge University Press (2000). DOI 10.1017/CBO9780511840531

48. Price, D., Laibe, G.: Two-phase mixtures in SPH - A new approach. Proceedings of 10th international SPHERIC workshop, Parma, Italy pp. 68-75 (2015)

49. Price, D.J., Laibe, G.: A fast and explicit algorithm for simulating the dynamics of small dust grains with smoothed particle hydrodynamics. Monthly Notices of the Royal Astronomical Society pp. 1-15 (2015). DOI 10.1093/mnras/stv996

50. Qu, X.L., Khezzar, L., Danciu, D., Labois, M., Lakehal, D.: Characterization of plunging liquid jets: a combined experimental and numerical investigation. International Journal of Multiphase Flow 37(7), 722-731 (2011). DOI 10.1016/j.ijmultiphaseflow.2011.02.006

51. Ren, B., Li, C., Yan, X., Ling, M.C., Bonet, J., Hu, S.M.: Multiple-fluid SPH simulation using a mixture model. ACM Transactions on Graphics 33, 171:1-171:11 (2014). DOI $10.1145 / 2645703$

52. Rezavand, M., Zhang, C., Hu, X.: Multi-phase simulation of highly violent flows using an SPH method based on a Riemann solver. In: Proceedings of the 14th International SPHERIC Workshop. Exeter, United Kingdom (2019)

53. Schiller, L., Naumann, Z.: A Drag Coefficient Correlation. Zeitschrift des Vereins Deutscher Ingenieure Zeitung 77, 318-320 (1935)

54. Shih, T.H., Liou, W.W., Shabbir, A., Yang, Z., Zhu, J.: A new $k-\epsilon$ eddy-viscosity model for high Reynolds number turbulent flows-model development and validation. Computers \& Fluids 24(3), 227-238 (1995). DOI 10.1016/0045-7930(94)00032-T

55. Simonin, O.: Prediction of the dispersed phase turbulence in particle-laden jets. ASME FED 121, 197-206 (1994)

56. Smoller, J.: Shock waves and reaction-diffusion equations. Second edition. Springer Verlag (1994)

57. Tafuni, A., Domínguez, J.M., Vacondio, R., Crespo, A.J.C.: A versatile algorithm for the treatment of open boundary conditions in Smoothed particle hydrodynamics GPU models. Computer Methods in Applied Mechanics and Engineering 342, 604-624 (2018). DOI 10.1016/j.cma.2018.08.004

58. Tran-Duc, T., Phan-Thien, N., Khoo, B.C.: A smoothed particle hydrodynamics (SPH) study of sediment dispersion on the seafloor. Physics of Fluids 29(8), 083302 (2017). DOI 10.1063/1.4993474

59. Vacondio, R., Rogers, B.D., Stansby, P.K., Mignosa, P.: SPH modelling of shallow flow with open boundaries for practical flood simulation. Journal of Hydraulic Engineering 138(6), 530-541 (2012). DOI 10.1061/(ASCE)HY.1943-7900.0000543

60. Valero, D., Bung, D.B.: Development of the interfacial air layer in the non-aerated region of high-velocity spillway flows. Instabilities growth, entrapped air and influence on the self-aeration onset. International Journal of Multiphase Flow 84, 66-74 (2016). DOI 10.1016/j.ijmultiphaseflow.2016.04.012

61. Violeau, D.: Dissipative forces for Lagrangian models in computational fluid dynamics and application to smoothed-particle hydrodynamics. Physical Review E 80 (2009). DOI 10.1103/PhysRevE.80.036705 
62. Violeau, D., Leroy, A.: On the maximum time step in weakly compressible SPH. Journal of Computational Physics 256, 388-415 (2014). DOI 10.1016/j.jcp.2013.09.001

63. Wendland, H.: Piecewise polynomial, positive definite and compactly supported radial functions of minimal degree. Advances in Computational Mathematics 4(1), 389-396 (1995). DOI 10.1007/BF02123482

64. Yan, X., Jiang, Y.T., Li, C.F., Martin, R.R., Hu, S.M.: Multiphase SPH simulation for interactive fluids and solids. ACM Transactions on Graphics 35(4) (2016). DOI $10.1145 / 2897824.2925897$ 